\title{
Discovery prospects of a light Higgs boson at the LHC in type-I 2HDM
}

\author{
Disha Bhatia, ${ }^{1, *}$ Ushoshi Maitra, ${ }^{1, \dagger}$ and Saurabh Niyogi ${ }^{2, \$}$ \\ ${ }^{1}$ Department of Theoretical Physics, Tata Institute of Fundamental Research, \\ Homi Bhabha Road, Colaba, Mumbai 400 005, India \\ ${ }^{2}$ Department of Physics and Astrophysics, University of Delhi, Delhi 110007, India
}

(Received 22 September 2017; published 22 March 2018)

\begin{abstract}
We present a comprehensive analysis of observing a light Higgs boson in the mass range $70-110 \mathrm{GeV}$ at the 13/14 TeV LHC, in the context of the type-I two-Higgs-doublet model. The decay of the light Higgs to a pair of bottom quarks is dominant in most parts of the parameter space, except in the fermiophobic limit. Here its decay to bosons (mainly a pair of photons) becomes important. We perform an extensive collider analysis for the $b \bar{b}$ and $\gamma \gamma$ final states. The light scalar is tagged in the highly boosted regimes for the $b \bar{b}$ mode to reduce the enormous QCD background. This decay can be observed with a few thousand $\mathrm{fb}^{-1}$ of integrated luminosity at the LHC. Near the fermiophobic limit, the decay of the light Higgs to a pair of photons can even be probed with a few hundred $\mathrm{fb}^{-1}$ of integrated luminosity at the LHC.
\end{abstract}

DOI: 10.1103/PhysRevD.97.055027

\section{INTRODUCTION}

The recently discovered scalar particle at the LHC $[1,2]$ closely resembles the Higgs boson conjectured in the Standard Model (SM), as its measured couplings with the gauge bosons and fermions are in reasonable agreement with the SM predictions [3]. However, the current measurements [3] still do not rule out the possibility of the observed particle belonging to an enlarged scalar sector of a beyond-the-SM scenario. Usually the additional scalars are considered to be heavy, and in some cases they are even decoupled from the low-energy effective theory. However, there may exist scenarios where some of the new physics particles are lighter than the observed Higgs. We explore this possibility in the context of the two-Higgs-doublet model (2HDM) at the $13 / 14 \mathrm{TeV}$ LHC.

The 2HDM is one of the simplest extensions of the SM with an additional scalar doublet charged under $S U(2)_{L}$. The generic structure of the $2 \mathrm{HDM}$ induces large flavorchanging neutral currents (FCNCs) at the tree level and consequently faces severe constraints from the experimental data. These FCNCs can be suppressed by imposing a discrete $Z_{2}$ symmetry. This classifies $2 \mathrm{HDM}$ into four categories: type I, type II, flipped, and lepton specific [4].

\footnotetext{
disha@theory.tifr.res.in †ushoshi@theory.tifr.res.in

*saurabhphys@gmail.com
}

Published by the American Physical Society under the terms of the Creative Commons Attribution 4.0 International license. Further distribution of this work must maintain attribution to the author(s) and the published article's title, journal citation, and DOI. Funded by SCOAP.
Any 2HDM model comprises of eight real scalar degrees of freedom. In the process of the spontaneous breaking of $S U(2)_{L} \times U(1)_{Y}$ symmetry, three of these eight fields generate masses for $W^{ \pm}$and $Z$ bosons, leaving behind five physical scalars, namely, a light $C P$-even Higgs $(h)$, a heavy $C P$-even Higgs $(H)$, a pseudoscalar $(A)$, and charged Higgs bosons $\left(H^{ \pm}\right)$.

The Higgs boson discovered at the LHC, being $C P$ even $[5,6]$, can be identified with any one of the $C P$-even states of the 2HDM. We are interested in the scenarios where the observed Higgs corresponds to the heavier $C P$-even scalar and $h$ is lighter than $125 \mathrm{GeV}$. The phenomenology of such a light Higgs has been thoroughly studied for all types of 2HDM. However, the constraints from vacuum stability, perturbativity, unitarity, electroweak precision measurements, flavor observables, and LHC Higgs searches are weakest for the type-I 2HDM [7-16]. We therefore focus on the type-I $2 \mathrm{HDM}$ for our analysis and study the discovery prospects of the light Higgs at the future runs of the LHC. ${ }^{1}$ We choose the mass range $70-110 \mathrm{GeV}$ to avoid decay of the observed $125 \mathrm{GeV}$ Higgs to a pair of onshell light Higgses, i.e., $H \rightarrow h h$. As a result, the bounds coming from the total decay width measurement of the observed scalar [31], the measurement of Higgs signal rate [3], and direct decay of the observed Higgs to a pair of light Higgses, i.e., $H \rightarrow h h$ [32] are irrelevant in our case

\footnotetext{
${ }^{1}$ The phenomenology of such a light $C P$-even scalar has also been studied in the context of various supersymmetric models; see Refs. [17-20] and references therein. Also see Refs. [21-30] for analyses where the lighter $C P$-even Higgs boson was identified with the observed scalar and the remaining scalars $\left(H, H^{ \pm}\right.$and $\left.A\right)$ were assumed to be heavy.
} 
(see Refs. [11,33] for an analysis with additional scalars lighter than $m_{h} / 2$ ).

To study the discovery prospects of the light $C P$-even scalar, a suitable choice of production and decay channels is essential. In our scenario, the light Higgs decays dominantly to $b \bar{b}$, except in the fermiophobic limit. Here its decay to bosons (mainly photons) becomes important. We therefore examine the light Higgs decays in the $b \bar{b}$ and $\gamma \gamma$ final states at the LHC. Note that the search for such lowmass scalars decaying to diphotons has already been performed at LHC Run-1 [34,35]. For the diphoton channel, we consider the production of the scalar through gluon fusion and in association with gauge bosons. The production of the light scalar in association with a gauge boson/top pair is considered for the $b \bar{b}$ mode. Owing to a clean environment, the diphoton final state is one of the favorite channels to search for new resonances at the LHC. In contrast, the $b \bar{b}$ state is plagued by the huge SM multijet backgrounds. Therefore, we consider the light Higgs in the boosted regimes for this channel, where the jet substructure techniques enable the efficient suppression of the SM backgrounds [36,37].

The paper is organized in the following manner. We begin with a brief introduction to the $2 \mathrm{HDM}$ in Sec. II, followed by a discussion of plausible channels which can be used to probe the light Higgs at the LHC in Sec. III. In $\mathrm{Sec}$. IV, we briefly review various constraints on the $2 \mathrm{HDM}$ parameter space arising from the LEP and LHC measurements, in the context of the type-I 2HDM. A dedicated collider analysis of the light Higgs in the allowed parameter space at the LHC is performed in Sec. V. Finally, in Sec. V E we summarize our results. Further, in Appendix A we discuss the light Higgs couplings to diphotons. In Appendix B the implications of the light charged Higgs boson on our results is analyzed, and in Appendix $\mathrm{C}$ the tagging methods used to reconstruct boosted objects are discussed. Finally, in Appendix D we tabulate the behavior of the total cross section of the selected modes with respect to the 2HDM parameters.

\section{2HDM: A BRIEF REVIEW}

The $Z_{2}$-symmetric $2 \mathrm{HDM}$ Lagrangian with two $S U(2)_{L}$ Higgs doublets $\left(\Phi_{1} \text { and } \Phi_{2}\right)^{2}$ can be parametrized as [4]

$$
\begin{aligned}
\mathcal{L}_{2 \mathrm{HDM}}= & \left(D_{\mu} \Phi_{1}\right)^{\dagger} D^{\mu} \Phi_{1}+\left(D_{\mu} \Phi_{2}\right)^{\dagger} D^{\mu} \Phi_{2}+\mathcal{L}_{\mathrm{Yuk}}\left(\Phi_{1}, \Phi_{2}\right) \\
& -V\left(\Phi_{1}, \Phi_{2}\right),
\end{aligned}
$$

where $\mathcal{L}_{\text {Yuk }}$ represents the Yukawa interactions and $V\left(\Phi_{1}, \Phi_{2}\right)$ is the scalar potential given as

\footnotetext{
${ }^{2}$ Under a $Z_{2}$ transformation, $\Phi_{1} \rightarrow \Phi_{1}$ and $\Phi_{2} \rightarrow-\Phi_{2}$.
}

$$
\begin{aligned}
V\left(\Phi_{1}, \Phi_{2}\right)= & m_{11}^{2} \Phi_{1}^{\dagger} \Phi_{1}+\frac{\lambda_{1}}{2}\left(\Phi_{1}^{\dagger} \Phi_{1}\right)^{2}+m_{22}^{2} \Phi_{2}^{\dagger} \Phi_{2} \\
& +\frac{\lambda_{2}}{2}\left(\Phi_{2}^{\dagger} \Phi_{2}\right)^{2}-\left[m_{12}^{2} \Phi_{1}^{\dagger} \Phi_{2}+\text { H.c. }\right] \\
& +\lambda_{3} \Phi_{1}^{\dagger} \Phi_{1} \Phi_{2}^{\dagger} \Phi_{2}+\lambda_{4} \Phi_{1}^{\dagger} \Phi_{2} \Phi_{2}^{\dagger} \Phi_{1} \\
& -\left[\frac{1}{2} \lambda_{5}\left(\Phi_{1}^{\dagger} \Phi_{2}\right)^{2}+\text { H.c. }\right] .
\end{aligned}
$$

Here $m_{12}$ is the soft $Z_{2}$-symmetry-breaking parameter. Note that in our analysis we have assumed $V\left(\Phi_{1}, \Phi_{2}\right)$ to be invariant under $C P$ (i.e., charge and parity transformations), and consequently the parameters of the scalar potential are real. The spontaneous breaking of $S U(2)_{L} \times U(1)_{Y}$ symmetry results in five physical scalar fields $h, H, A$, and $H^{ \pm}$ (with masses $m_{h}, m_{H}, m_{A}$, and $m_{H^{ \pm}}$, respectively) and three Goldstone bosons $G$ and $G^{ \pm}$, which appear as the longitudinal modes for $Z$ and $W^{ \pm}$bosons. The mass spectra of the particles are obtained by minimizing the scalar potential $V\left(\Phi_{1}, \Phi_{2}\right)$ in Eq. (2).

The doublets in terms of the physical fields and the Goldstone bosons can be expressed as

$$
\begin{gathered}
\Phi_{1}=\left(\begin{array}{c}
G^{+} \cos \beta+H^{+} \sin \beta \\
\frac{1}{\sqrt{2}}\left[h \sin \alpha-H \cos \alpha+i(G \cos \beta+A \sin \beta)+v_{1}\right]
\end{array}\right), \\
\Phi_{2}=\left(\begin{array}{c}
G^{+} \sin \beta-H^{+} \cos \beta \\
\frac{1}{\sqrt{2}}\left[-h \cos \alpha-H \sin \alpha+i(G \sin \beta-A \cos \beta)+v_{2}\right]
\end{array}\right),
\end{gathered}
$$

where $\alpha$ and $\beta$ are the rotation angles which diagonalize the mass matrices for the neutral $C P$-even Higgs and the charged Higgs/ $C P$-odd Higgs, respectively. The parameters of the scalar potential $\left(m_{11}, m_{22}, \lambda_{i}\right)$ can be expressed in terms of the rotation angles $(\alpha, \beta)$, the $Z_{2}$-symmetrybreaking parameter $\left(m_{12}\right)$, and the masses of the scalars $\left(m_{h}, m_{H}, m_{A}, m_{H}^{ \pm}\right)$as [4]

$$
\begin{aligned}
m_{11}^{2}= & \frac{1}{4}\left(m_{h}^{2}+m_{H}^{2}-4 m_{12}^{2} \tan \beta\right. \\
& \left.+\left(m_{H}^{2}-m_{h}^{2}\right) \sec \beta \cos (2 \alpha-\beta)\right), \\
m_{22}^{2}= & \frac{1}{4}\left(m_{h}^{2}+m_{H}^{2}-4 m_{12}^{2} \cot \beta\right. \\
& \left.+\left(m_{H}^{2}-m_{h}^{2}\right) \csc \beta \sin (2 \alpha-\beta)\right), \\
\lambda_{1}= & \frac{1}{2 v^{2}} \sec ^{2} \beta\left(m_{h}^{2}+m_{H}^{2}+\left(m_{H}^{2}-m_{h}^{2}\right) \cos 2 \alpha\right. \\
& \left.-2 m_{12}^{2} \tan \beta\right),
\end{aligned}
$$

$\lambda_{2}=\frac{1}{2 v^{2}} \csc ^{2} \beta\left(m_{h}^{2}+m_{H}^{2}-\left(m_{H}^{2}-m_{h}^{2}\right) \cos 2 \alpha-2 m_{12}^{2} \cot \beta\right)$, 
$\lambda_{3}=\frac{1}{v^{2}} \csc 2 \beta\left(-2 m_{12}^{2}+\left(m_{H}^{2}-m_{h}^{2}\right) \sin 2 \alpha+2 m_{H^{ \pm}}^{2} \sin 2 \beta\right)$,

$$
\begin{gathered}
\lambda_{4}=\frac{1}{v^{2}}\left(m_{A}^{2}-2 m_{H^{ \pm}}^{2}+m_{12}^{2} \csc \beta \sec \beta\right), \\
\lambda_{5}=\frac{1}{v^{2}}\left(m_{12}^{2} \csc \beta \sec \beta-m_{A}^{2}\right), \\
v_{1}=v \cos \beta, \quad \text { and } \quad v_{2}=v \sin \beta
\end{gathered}
$$

The couplings $\lambda_{i}(i=1,5)$ are constrained by the perturbativity, vacuum stability [38], and unitarity [39] bounds, which in turn restrict the allowed values of the scalar masses for given values of $\alpha$ and $\beta[4,40]$. The masses of the additional scalars are also constrained from the well-measured flavor and electroweak observables [41-43]. The combined effect of these constraints on the 2HDM parameter space is discussed in Appendix B in the context of the type-I 2HDM. Note that the free parameter $\alpha$ remains unaffected after imposing the above constraints (see Fig. 12 in Appendix B).

In our analysis, we identify the heavier $C P$-even Higgs with the discovered scalar by fixing $m_{H}=125 \mathrm{GeV}$ and study the phenomenology of the light $C P$-even scalar $h$. At this stage we have the following free parameters: $\alpha, \beta, m_{H^{ \pm}}$, $m_{A}, m_{12}$ and $m_{h}$. However, we confine ourselves to that part of the allowed parameter space where the masses of the charged and pseudoscalar Higgs bosons are heavy [i.e., $\mathcal{O}(500) \mathrm{GeV}]$ and thus do not affect our analysis. The $\mathrm{Z}_{2}$-breaking parameter in this case becomes irrelevant for the light Higgs phenomenology and can be suitably chosen to have any value less than $100 \mathrm{GeV}$ (see Appendix B). Note that although we have chosen the charged Higgs to be heavy for most of our analysis, we do analyze the implications of having a low-mass charged scalar in Appendix B.

We now discuss the couplings of the scalar particles with fermions and gauge bosons. In the type-I 2HDM, fermions couple only to one of the doublets, i.e., $\Phi_{2}$ and $\mathcal{L}_{\text {Yuk }}$ is given as

$$
\begin{aligned}
\mathcal{L}_{\text {Yuk }}^{\text {type-I }}= & \overline{Q_{L}} \mathcal{Y}^{d} \Phi_{2} d_{R}+\overline{Q_{L}} \mathcal{Y}^{u} \Phi_{2}^{c} u_{R}+\overline{Q_{L}} \mathcal{Y}^{e} \Phi_{2}^{c} e_{R}+\text { H.c., } \\
= & -\sum_{f=u, d, \ell} \frac{m_{f}}{v}\left(\xi_{h}^{f} \bar{f} f h+\xi_{H}^{f} \bar{f} f H-i \xi_{A}^{f} \bar{f} \gamma_{5} f A\right) \\
& -\frac{\sqrt{2} V_{u d}}{v} \bar{u}\left(m_{u} \xi_{A}^{u} P_{L}+m_{d} \xi_{A}^{d} P_{R}\right) d H^{+} \\
& -\frac{\sqrt{2} m_{\ell}}{v} \xi_{A}^{\ell} \bar{\nu} P_{R} \ell H^{+}+\text {H.c. },
\end{aligned}
$$

where $\mathcal{Y}^{u, d, e}$ are $3 \times 3$ Yukawa matrices, $V_{u d}$ is the Cabibbo-Kobayashi-Maskawa matrix element, $m_{f}$ is the mass of a fermion $(f)$, and

$$
\begin{aligned}
\xi_{h}^{u, d, \ell} & =\cos \alpha / \sin \beta, \quad \xi_{H}^{u, d, \ell}=\sin \alpha / \sin \beta, \\
\xi_{A}^{u} & =\cot \beta, \quad \xi_{A}^{d, \ell}=-\cot \beta .
\end{aligned}
$$

We list some of the couplings of gauge bosons with scalars that are relevant for our analysis (see Refs. $[44,45]$ for a complete list):

$$
\begin{aligned}
\mathcal{L}_{\text {Gauge-int }}= & \frac{m_{Z}^{2}}{v} \xi_{h}^{V} Z_{\mu} Z^{\mu} h+\frac{m_{Z}^{2}}{v} \xi_{H}^{V} Z_{\mu} Z^{\mu} H+2 \frac{m_{W}^{2}}{v} \xi_{h}^{V} W_{\mu} W^{\mu} h \\
& +2 \frac{m_{W}^{2}}{v} \xi_{H}^{V} W_{\mu} W^{\mu} H+\frac{\alpha_{e m}}{8 \pi v} \xi_{h}^{\gamma} h F_{\mu \nu} F^{\mu \nu} \\
& +\frac{\alpha_{e m}}{8 \pi v} \xi_{H}^{\gamma} H F_{\mu \nu} F^{\mu \nu}
\end{aligned}
$$

where

$$
\xi_{h}^{V}=\sin (\beta-\alpha), \quad \xi_{H}^{V}=\cos (\beta-\alpha),
$$

and the expressions for $\xi_{h}^{\gamma}$ and $\xi_{H}^{\gamma}$ are listed in Appendix A. Based on the above couplings of the scalars with fermions and gauge bosons, two interesting limits arise.

(1) Alignment limit $(\alpha \rightarrow \beta)$ : Here the couplings of the heavier $C P$-even Higgs exactly match those of the SM Higgs.

(2) Fermiophobic limit ( $\alpha \rightarrow \pi / 2)$ : In this limit, the treelevel couplings of the light Higgs with fermions $\left(\xi_{h}^{f}\right)$ vanish [see Eq. (13)] and its loop-induced couplings with fermions are also negligible. The light Higgs in this case behaves as a fermiophobic scalar.

We shall see later that these limits have interesting implications in our analysis. As an aside, note that the condition $\alpha \equiv \beta \rightarrow \pi / 2$ corresponds to the case where the alignment and fermiophobic limits occurs simultaneously. In this case, the couplings of the light Higgs with both fermions and gauge bosons vanish [see Eqs. (13) and (15)] and the type-I 2HDM maps to the inert 2HDM model.

After discussing the couplings of the light Higgs boson, we are now in a position to predict its phenomenological consequences. In the next section, we identify the promising channels which could be useful in probing the light Higgs at the LHC. Keeping a large QCD background in mind, a suitable choice of the production channel and decay mode would be essential for the discovery of a light scalar like the SM Higgs.

\section{PROMISING CHANNELS TO EXPLORE AT THE LHC}

The light Higgs boson, just like the SM Higgs, can be produced at the LHC via gluon fusion ( $\mathrm{ggF}$ ), vector-boson fusion (VBF), and in association with SM gauge bosons $(\mathrm{V} h)$, as well as with a top pair $(t \bar{t} h)$. The ratio of the production cross section of the light Higgs and that of the SM-like Higgs $\left(h_{\mathrm{SM}}\right)$ as a function of $\alpha$ is plotted in the left panel of Fig. 1. Here, by "SM-like Higgs" we mean a 

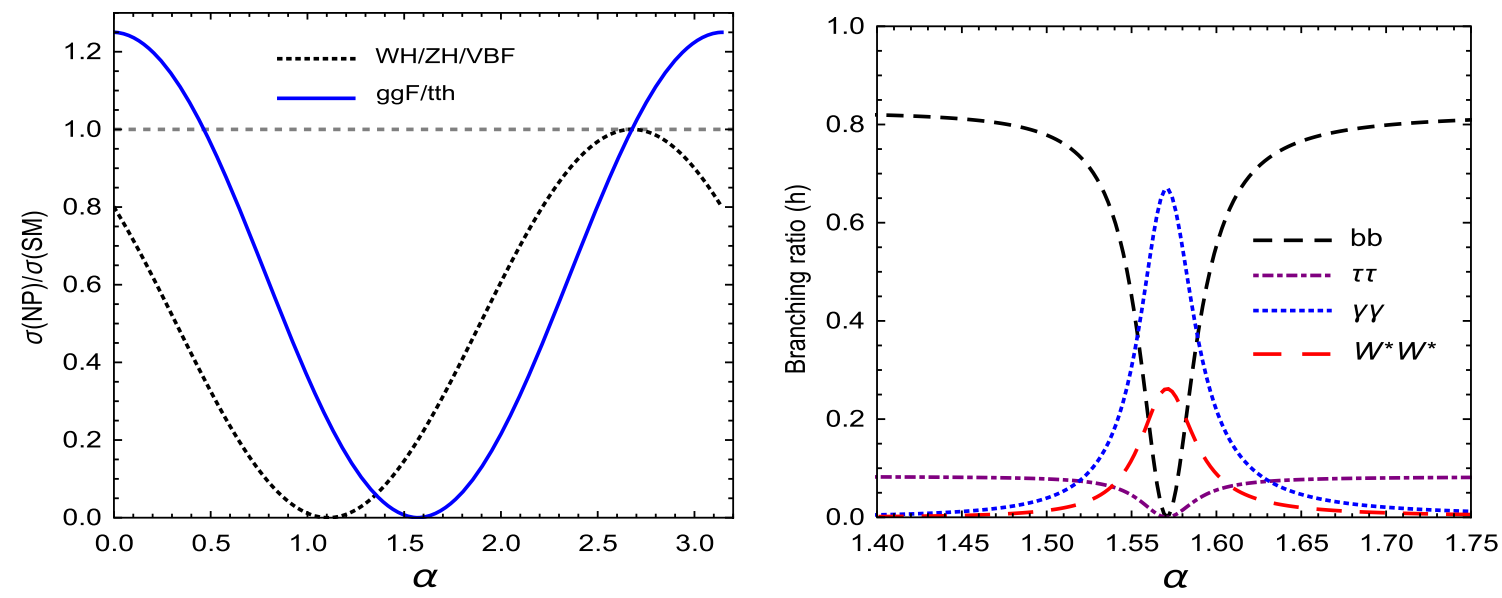

FIG. 1. A representative plot for $m_{h}=80 \mathrm{GeV}$ and $\tan \beta=2$. The left panel illustrates the variation of the ratio of the cross sections of the light Higgs $(h)$ and the SM-like Higgs $\left(h_{\mathrm{SM}}\right)$ with $\alpha$. The right panel shows the branching ratios of the light Higgs as a function of $\alpha$ (the range of $\alpha$ is restricted near $\pi / 2$ to signify the behavior around the fermiophobic limit). Note that the $\mathrm{Vh} / \mathrm{VBF}$ and $\mathrm{ggF} / \bar{t} \bar{t} h$ production modes are able to probe a similar parameter space. In a similar fashion, the variations of the $b \bar{b}$ and $\tau \bar{\tau}$ decay modes are identical with $\alpha$.

hypothetical scalar whose couplings are exactly the same as those of the SM Higgs but whose mass is equal to that of the light Higgs, i.e., $m_{h_{\mathrm{SM}}}=m_{h}$. Note that $m_{h}$ is chosen to be $80 \mathrm{GeV}$ in Fig. 1 for illustrative purposes. The gluon fusion as well as the $t \bar{t} h$ production cross section of the light Higgs in the type-I model scale as $\left(\xi_{h}^{f}\right)^{2}$ with respect to the SM-like Higgs. Similarly, the cross sections for the light Higgs produced in association with gauge bosons or through vector-boson fusion scale as $\left(\xi_{h}^{V}\right)^{2}$. Therefore, the ordinate in Fig. 1 essentially shows the variation of $\left(\xi_{h}^{i}\right)^{2}$ against $\alpha$. The scaling has been illustrated for $\tan \beta=$ 2 in Fig. 1. It can be seen from Eqs. (13) and (15) that for large values of $\tan \beta$, all production channels scale identically as $\cos ^{2} \alpha$.

The right panel of Fig. 1 represents various branching fractions of the light Higgs again as a function of $\alpha$. In most of the parameter space, the light Higgs decays dominantly to a pair of bottom quarks. However, near $\alpha \rightarrow \pi / 2$ (the fermiophobic limit) it decays maximally to a pair of gauge bosons. We therefore choose $b \bar{b}$ and $\gamma \gamma$ as the light Higgs decay modes for our analysis. We must stress that the branching ratio of $h$ to a $\tau$ pair is also significant $(\sim 10 \%)$. Since the parameter space probed by it is similar to that of $b \bar{b}$, we restrict ourselves to the analysis of $b \bar{b}$ in this manuscript.

Now our task is to determine the suitable production mode for a light Higgs decaying to a pair of bottom quarks and photons. Note that analyzing the $b \bar{b}$ channel in the $\mathrm{ggF}$ or VBF mode is challenging due to the presence of a large QCD background. However, the presence of a lepton(s) in addition to $b \bar{b}$ in $V h$ or $t \bar{t} h$ production modes could help to suppress these backgrounds. Hence, we choose light Higgs production in association with a $W$ boson and top pair for $b \bar{b}$ analysis. ${ }^{3}$ On the other hand, the diphoton channel is one of the cleanest probes for discovering new resonances at the LHC. This channel also comes with the additional advantage of enhanced sensitivity near the fermiophobic limit in the type-I 2HDM. In this limit, i.e., $\alpha \rightarrow \pi / 2$ the decay of $h$ to $\gamma \gamma$ becomes prominent and can only be probed through the Vh/VBF production mode, as shown in Fig. 1. We have considered only the $W h$ process in our analysis as the parameter space probed by VBF and $Z h$ are exactly the same as that of $W h$. The diphoton channel can also be used to probe regions away from the fermiophobic limit through the $g g F / t \bar{t} h$ production mode. Since the production cross section of $t \bar{t} h$ is roughly 100 times smaller than that of $\mathrm{ggF}$, we have not considered this for the diphoton analysis. To summarize, we have chosen the following channels ${ }^{4}$ for probing the light Higgs at the LHC:

Channel 1: $p p \rightarrow h \rightarrow \gamma \gamma$.

Channel 2: $p p \rightarrow W h \rightarrow W \gamma \gamma$.

Channel 3: $p p \rightarrow W h \rightarrow b \bar{b}$.

Channel 4: $p p \rightarrow t \bar{t} h \rightarrow t \bar{t} b \bar{b}$.

The phenomenological consequences of these channels will be examined in Sec. V.

\section{EXPERIMENTAL CONSTRAINTS}

In this section, we discuss the experimental constraints on the 2HDM parameter space (i.e., $\alpha, \tan \beta$, and $m_{h}$ ) from the observed Higgs signal strength measurements and the

\footnotetext{
${ }^{3}$ The $Z h$ production mode is neglected as the leptonic branching ratio in the case where $Z$ is smaller than $W$ and the parameter space probed by $W h$ and $Z h$ are exactly the same.

${ }^{4}$ The behavior of the total cross section with respect to $\alpha$ and $\beta$ corresponding to the four selected channels is discussed in Appendix D.
} 
direct searches for light scalars at LEP and LHC. In our analysis, we have varied $\alpha$ in its full range, i.e., $[0: \pi]$ and $\tan \beta$ in the restricted range of $[1: 10]$. While the lower value of $\tan \beta$ is chosen to account for the constraints from the flavor observables (as discussed in Appendix B), the higher value is restricted to 10 for interesting phenomenology. ${ }^{5}$ The organization of this section is as follows. In Secs. IV A and IV B we discuss the individual constraints from the signal strength measurements and the direct searches for light scalars, respectively. Towards end of Sec. IV B, the combined effect of the above constraints on the parameter space is presented.

\section{A. LHC constraints: Signal strength measurements of the $125 \mathrm{GeV}$ Higgs}

Since we have identified the heavier $C P$-even Higgs with the observed Higgs boson, its couplings with fermions and gauge bosons-which are different from that of the SM by the factors $\xi_{H}^{f}, \xi_{H}^{V}, \xi_{H}^{\gamma}$-get constrained by the signal strength measurements [3]. If the observed Higgs is produced through channel $i$ and decays to $j$, then the signal strength $\left(\mu_{j}^{i}\right)$ (assuming the narrow-width approximation) is defined as $[3,46]$

$\mu_{j}^{i}=\frac{\sigma(i \rightarrow H)}{\sigma\left(i \rightarrow H_{\mathrm{SM}}\right)} \times \frac{\mathrm{BR}(H \rightarrow j)}{\mathrm{BR}\left(H_{\mathrm{SM}} \rightarrow j\right)}=\xi_{H}^{\mathrm{prod}, i} \times \xi_{H}^{\mathrm{dec}}, \frac{\Gamma_{H_{\mathrm{SM}}}^{\mathrm{tot}}}{\Gamma_{H}^{\mathrm{tot}}}$,

where

$$
\begin{aligned}
\xi_{H}^{\mathrm{prod}, i} & =\frac{\sigma(i \rightarrow H)}{\sigma\left(i \rightarrow H_{\mathrm{SM}}\right)}, \quad \xi_{H}^{\mathrm{decay}, j}=\frac{\Gamma(H \rightarrow j)}{\Gamma\left(H_{\mathrm{SM}} \rightarrow j\right)}, \\
\Gamma_{H}^{\mathrm{tot}} & =\sum_{k} \xi_{H}^{\text {decay }, k} \Gamma_{H}^{k, \mathrm{SM}} .
\end{aligned}
$$

In Table I we list the production and decay scaling factors for the observed Higgs. Note that these factors are exact only at the leading order. However, the deviations after including the higher-order corrections are small [14] and hence are neglected in the analyses.

The measured signal strengths, i.e., $\left(\mu_{j}^{i}\right)^{\exp }$ used in our analysis are listed in Table II. Note that these measurements do not constrain the mass of the light Higgs due to the absence of the $H \rightarrow h h$ decay mode as we have considered $m_{h}>m_{H} / 2$ in our analysis. Hence the parameters that are constrained by Higgs signal strength measurements are $\alpha$ and $\beta$. In Fig. 2, we present the allowed regions in the $(\sin (\beta-\alpha), \tan \beta)$ plane after incorporating constraints from the Higgs signal strength measurements. These regions are determined by allowing individual $\mu_{j}^{i}$ predicted

\footnotetext{
${ }^{5}$ With an increase in $\tan \beta$, the couplings of the light Higgs in the type-I 2HDM with fermions decrease, and for gauge bosons they become independent of $\beta$.
}

TABLE I. Scaling factors for the production and decay processes. See Eqs. (13), (14), and (A3) for the definitions of $\xi_{H}^{f}, \xi_{H}^{V}$, and $\xi_{H}^{\gamma}$ respectively.

\begin{tabular}{lcccccc}
\hline \hline Production & $g g F / t \bar{t} H$ & $\mathrm{VBF} / V H$ & Decay & $f \bar{f}$ & $V V^{*}$ & $\gamma \gamma$ \\
\hline$\xi_{H}^{\text {prod }}$ & $\left(\xi_{H}^{f}\right)^{2}$ & $\left(\xi_{H}^{V}\right)^{2}$ & $\xi_{H}^{\text {decay }}$ & $\left(\xi_{H}^{f}\right)^{2}$ & $\left(\xi_{H}^{V}\right)^{2}$ & $\left(\xi_{H}^{\gamma}\right)^{2}$ \\
\hline \hline
\end{tabular}

TABLE II. The combined measured values of $\left(\mu_{j}^{i}\right)^{\exp }$ from ATLAS and CMS using 7 and $8 \mathrm{TeV}$ data [3] used in our analysis. The allowed regions in the parameter space are determined by allowing individual $\mu_{j}^{i}$ predicted in the type-I $2 \mathrm{HDM}$ to lie within $\pm 2 \sigma$ from the central values of the measured signal strengths, i.e., $\left(\mu_{j}^{i}\right)^{\exp }$.

\begin{tabular}{lccc}
\hline \hline $\begin{array}{l}\text { Signal } \\
\text { strength } \\
\left(\mu_{j}^{\text {ggF }}\right)^{\exp }\end{array}$ & $\begin{array}{c}\text { ATLAS-CMS } \\
(7-8 \mathrm{TeV}) \\
(\text { combined })\end{array}$ & $\begin{array}{c}\text { Signal } \\
\text { strength } \\
\left(\mu_{j}^{\mathrm{VBF}}\right)\end{array}$ & $\begin{array}{c}\text { exp } \\
\text { ATLAS-CMS } \\
(7-8 \mathrm{TeV}) \\
(\text { combined })\end{array}$ \\
\hline$\mu_{\gamma \gamma}^{\mathrm{ggF}}$ & $1.10_{-0.22}^{+0.23}$ & $\mu_{\gamma \gamma}^{\mathrm{VBF}}$ & $1.3_{-0.5}^{+0.5}$ \\
$\mu_{\mathrm{ZZ}}^{\mathrm{ggF}}$ & $1.13_{-0.31}^{+0.34}$ & $\mu_{\mathrm{ZZ}}^{\mathrm{VBF}}$ & $0.1_{-0.6}^{+1.1}$ \\
$\mu_{W W}^{\mathrm{ggF}}$ & $0.84_{-0.17}^{+0.17}$ & $\mu_{W W}^{\mathrm{VBF}}$ & $1.2_{-0.4}^{+0.4}$ \\
$\mu_{\tau \bar{\tau}}^{\mathrm{ggF}}$ & $1.0_{-0.6}^{+0.6}$ & $\mu_{\tau \bar{\tau}}^{\mathrm{VBF}}$ & $1.3_{-0.4}^{+0.4}$ \\
\hline \hline
\end{tabular}

in the type-I $2 \mathrm{HDM}$ to lie within $\pm 2 \sigma$ of the central values of $\left(\mu_{j}^{i}\right)^{\exp }$ obtained from the combined ATLAS and CMS 7 and $8 \mathrm{TeV}$ data [3]. However, we must mention that we have not employed the $\chi^{2}$ minimization technique while deriving such allowed regions using data.

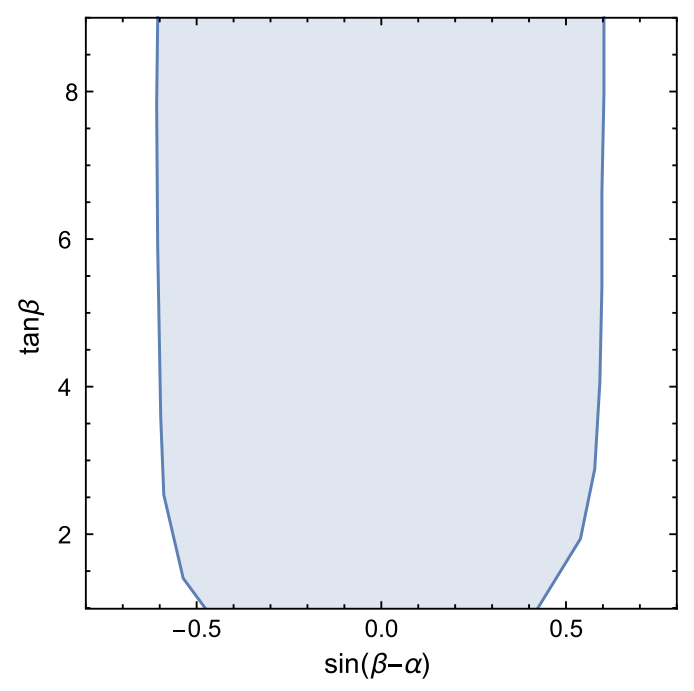

FIG. 2. The allowed region (shaded in blue) is determined by allowing individual $\mu_{i}^{j}$ predicted in the type-I 2HDM to lie within $\pm 2 \sigma$ of the central values of $\left(\mu_{j}^{i}\right)$ exp obtained from the combined ATLAS and CMS 7 and $8 \mathrm{TeV}$ data [3]. The signal strengths considered for the analysis are listed in Table II. Note that we have not employed the $\chi^{2}$ minimization technique in our analysis for determining the allowed regions of the parameter space. 
We now discuss the qualitative features of Fig. 2. The constraints from the Higgs signal strength measurements force us to remain close to the alignment limit as the heavier $C P$-even Higgs here behaves exactly like the SM Higgs. In Fig. 2 , one could notice that for $\tan \beta \approx 1$, negative values of $\sin (\beta-\alpha)$ are slightly less constrained than positive ones. In this region, $\sin (\beta-\alpha)>0$ implies $\alpha<\pi / 4$ and $\sin (\beta-$ $\alpha)<0$ implies $\alpha>\pi / 4$. As a result, the Yukawa couplings of the SM-like Higgs [which scale as $\left(\xi_{H}^{f}\right)$ ] decrease for increasing positive values of $\sin (\beta-\alpha)$. Therefore, the signal strength $\mu_{j}^{g g F}$ (which depends on $\xi_{H}^{f}$ ) drops quickly below the allowed range for positive values of $\sin (\beta-\alpha)$, making this region relatively more constrained. For larger values of $\tan \beta, \sin (\beta-\alpha)$ is approximately equal to $\cos \alpha$. Hence, the allowed region in Fig. 2 becomes symmetric in $\sin (\beta-\alpha)$ as well as independent of $\tan \beta$. Although we have plotted the effect of signal strength constraints in the $(\sin (\beta-\alpha), \tan \beta)$ plane, this can be easily translated to the $(\alpha, \tan \beta)$ plane. The net effect is only to restrict the allowed range of $\alpha$ to be less than $\pi$ for a given value of $\tan \beta$.

\section{B. Light Higgs direct search bounds}

A $C P$-even scalar has been searched for in the channel $e^{+} e^{-} \rightarrow Z h$ [47] at LEP. The cross section for this process scales as $\left(\xi_{h}^{V}\right)^{2}$, i.e., $\sin ^{2}(\beta-\alpha)$. The absence of any excess in this process has severely constrained $|\sin (\beta-\alpha)|$. In the left panel of Fig. 3, we list the upper limits on $|\sin (\beta-\alpha)|$ at $95 \%$ C.L. for different masses of the light Higgs [47].

Note that as the center-of-mass energy at LEP was limited to $209 \mathrm{GeV}$, the production cross section of the light Higgs for the heavier masses faced severe phase-space suppression. As a result, these masses are less constrained by the LEP data. In the right panel of Fig. 3, we project the LEP bounds listed in the left panel onto the allowed regions at $95 \%$ C.L. in the $(\alpha, \tan \beta)$ plane for $m_{h}=90 \mathrm{GeV}$ (pink) and $m_{h}=100 \mathrm{GeV}$ (blue) for illustrative purposes. Note that the LEP constraint- just like the Higgs signal strength -restricts the allowed range of $\alpha$ to be less than $\pi$, for a given $\tan \beta$ and $m_{h}$. We must mention that the Tevatron also searched for such a light Higgs in the $V h$ production mode [48]. However, the Tevatron bounds are much less stringent than LEP and hence are not considered in the analysis.

LEP has also searched for a $C P$-odd scalar in the process $e^{+} e^{-} \rightarrow h A \quad[49,50]$. This search is complimentary to $e^{+} e^{-} \rightarrow h Z$ as the former depends on $\cos ^{2}(\beta-\alpha)$ and the latter on $\sin ^{2}(\beta-\alpha)$. The null results in both production modes significantly constrain both $\sin (\beta-\alpha)$ and $\cos (\beta-\alpha)$ and require them to be much less than unity. If both $h$ and $A$ are light at the same time such that $m_{A}+m_{h}<209 \mathrm{GeV}$, then the combined direct search constraints of $h$ and $A$ rule out a significant part of the parameter space including the regions which satisfy the alignment limit. Therefore, our choice of demanding a heavy pseudoscalar is in sync with the requirement of a light Higgs.

Both the ATLAS and CMS Collaborations have searched for additional light scalars in the diphoton final state [34,35,51]. CMS has placed 95\% C.L. upper bounds on the total cross section for a light scalar decaying to $\gamma \gamma$ for the production modes ggF $+t \bar{t} h$ and $\mathrm{VBF}+V h$. On the other hand, ATLAS provides an inclusive bound for the combination of all of the production modes. To understand the effect of these measurements on the parameter space of the 2HDM, let us note the behavior of the total cross section of the light Higgs decaying to a pair of photons. We now know that the light Higgs branching ratio to a pair of photons is large near the fermiophobic limit and could be probed in the $\mathrm{VBF}+V h$ production mode. However, in this case, the total cross section, i.e., $\sigma \times \mathrm{BR}$ is large only

\begin{tabular}{||c|c||}
\hline \hline $\begin{array}{c}\text { Light Higgs } \\
\text { mass }\end{array}$ & $\begin{array}{c}\text { Maximum allowed } \\
|\sin (\beta-\alpha)| \text { from LEP }\end{array}$ \\
\hline $70 \mathrm{GeV}$ & 0.165 \\
\hline $80 \mathrm{GeV}$ & 0.21 \\
\hline $90 \mathrm{GeV}$ & 0.39 \\
\hline $100 \mathrm{GeV}$ & 0.49 \\
\hline $110 \mathrm{GeV}$ & 0.54 \\
\hline \hline
\end{tabular}

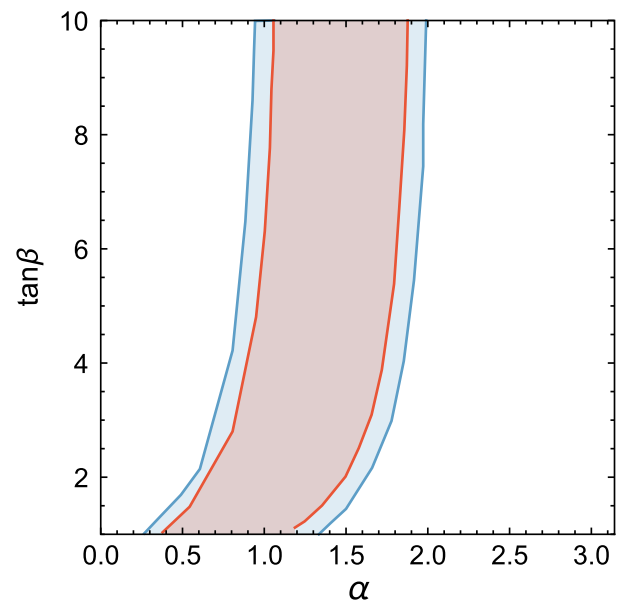

FIG. 3. In the left panel we list the upper bounds on $|\sin (\beta-\alpha)|$ at 95\% C.L. obtained from the LEP direct search measurements [47] for different light Higgs masses. In the right panel we translate these bounds into allowed regions at 95\% C.L. in the $(\alpha$, tan $\beta)$ plane. The regions shaded in blue (pink) correspond to $m_{h}=90(100) \mathrm{GeV}$. Note that the effect of the LEP constraint limits the allowed range of $\alpha$ for a given $\tan \beta$ and light Higgs mass. 


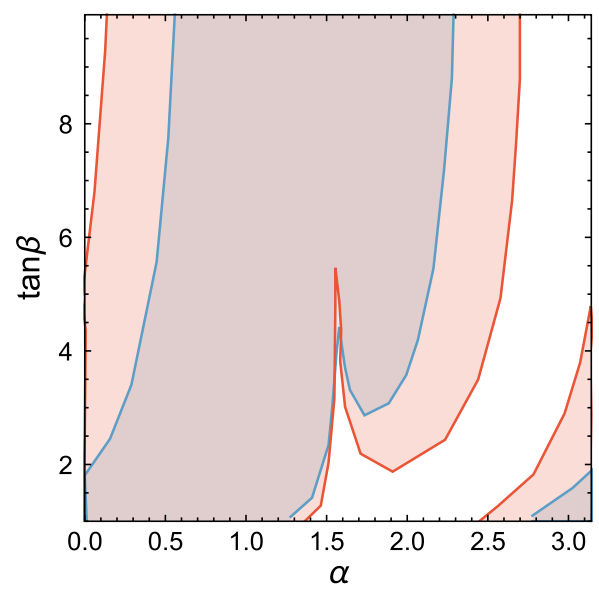

FIG. 4. We demonstrate the allowed regions by incorporating constraints only from the light scalar searches at the LHC $[34,35,51]$ in the $(\alpha, \tan \beta)$ plane for $m_{h}=90 \mathrm{GeV}$ (pink band) and $100 \mathrm{GeV}$ (blue band). The masses have been chosen for illustrative purposes, as before.

for smaller values of $\tan \beta$ and tends to zero for larger values of $\tan \beta$ (see Table VII in Appendix D). For regions away from the fermiophobic limit, although the branching ratio of the light Higgs to a pair of photons is not large, this decay could still be probed in the ggF mode owing to its large production cross section.

The effect of the LHC direct detection constraints $[34,35,51]$ are displayed in Fig. 4, where we plot the allowed parameter space in the $(\alpha, \tan \beta)$ plane for $m_{h}=$ $90 \mathrm{GeV}$ (pink band) and $100 \mathrm{GeV}$ (blue band). The masses have been chosen for illustrative purposes, as before. The combined bounds from ATLAS and CMS near the fermiophobic limit are sensitive only to the $\mathrm{VBF}+V h$ production mode, where the total cross section is large for smaller $\tan \beta$ values. Consequently, this region gets severely constrained and results in a wedge-like exclusion around $\alpha \approx \pi / 2$, as can be seen in Fig. 4. For regions away from the fermiophobic limit, the combined constraints from ATLAS and CMS [34,35,51] are far more stringent for $m_{h}=100 \mathrm{GeV}$ than for $90 \mathrm{GeV}$, and hence they rule out a significant part of the parameter space for $m_{h}=100 \mathrm{GeV}$.

Now we combine the individual constraints from the Higgs signal strength measurements [3] and the direct searches for the low-mass scalars at LEP and LHC $[34,35,47,51]$. The results are shown in Fig. 5 in the $(\alpha, \tan \beta)$ plane for $m_{h}=90 \mathrm{GeV}$ (pink band) and $100 \mathrm{GeV}$ (blue band). As already noted, the effect of the direct detection constraints from LEP and the Higgs signal strength measurements is to restrict the allowed range of $\alpha$ to be less than $\pi$. In our case, the LEP constraints are far more stringent than those arising from the Higgs signal strength. In Fig. 5, we can see that the allowed range of $\alpha$ increases as the light Higgs mass increases. This happens due to the relaxed LEP constraints for a heavier light Higgs mass (see left panel of Fig. 3). In contrast, the direct search

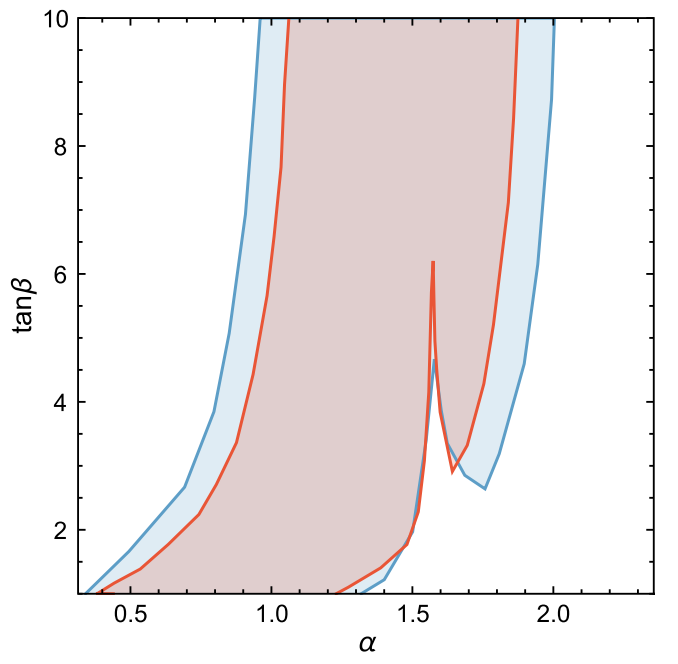

FIG. 5. The net allowed parameter space for the type-I 2HDM in the $(\alpha, \tan \beta)$ plane for $m_{h}=90 \mathrm{GeV}$ (pink band) and $100 \mathrm{GeV}$ (blue band) after combing measurements from the Higgs signal strength [3] and the direct searches for light scalar at LEP and LHC $[34,35,47,51]$. The wedge-like disallowed region around $\alpha \approx \pi / 2$ arises from the direct searches for the light Higgs decaying to a pair of photons at the LHC. This constraint gets relaxed for larger values of $\tan \beta$ and with increasing mass of the light Higgs due to suppression in the production cross section.

for a light Higgs at the LHC rules out a wedge-like region around the fermiophobic limit and some regions away from the fermiophobic limit. However, the constraints for the latter from the LHC are much weaker than the LEP constraints and consequently are masked in the combination (see Fig. 5). Note that the LHC constraint around the fermiophobic limit gets relaxed for larger values of $\tan \beta$ and with increasing mass of the light Higgs due to suppression in the production cross section.

\section{FUTURE PROSPECTS AT LHC RUN-2}

In this section, we discuss the prospects of observing a light Higgs boson in the following channels: $p p \rightarrow h \rightarrow \gamma \gamma, p p \rightarrow W h \rightarrow W \gamma \gamma, p p \rightarrow W h \rightarrow b \bar{b}$, and $p p \rightarrow t \bar{t} h \rightarrow t \bar{t} b \bar{b}$. The signal and background processes ${ }^{6}$ corresponding to each channel are generated using the event generators MADGRAPH [52] or PYTHIA-8 [53,54] (depending on the number of final-state hard particles at the parton level) with the NN23LO1 [55] parton distribution function. The generated events are then showered and hadronized using PYTHIA-8. Note that the collider analysis has been carried out in PYTHIA. We have not performed any

\footnotetext{
${ }^{6}$ Note that there are two types of backgrounds associated with a particular signal topology: reducible and irreducible. While the irreducible backgrounds consist of exactly the same final states, the reducible backgrounds are somewhat different and contribute to a particular signal topology because of the misidentification of objects.
} 
detector simulation in the analysis. We now describe the basic cuts used in our analysis.

(1) A minimum cut of $20 \mathrm{GeV}$ is imposed on the transverse momentum of photons, electrons, muons, and missing energy.

(2) Owing to the finite resolution of the electromagnetic calorimeter, photons and electrons (muons) are accepted for further analysis if their pseudorapidities are less than 2.5 (2.7).

(3) Photons and leptons (electrons and muons) are required to be isolated, meaning free from the dominant jet activity in their nearby regions.

(4) In experiments, there is a typical 5\% probability for an electron to fake a photon, due to track mismeasurements. Since this feature is not present in PYTHIA, we take this into account in our analysis with the help of a random number. We randomly select $5 \%$ events, where an electron is mistagged as a photon.

(5) The hadrons are clustered into jets with jet radius $R=0.4$ using the anti- $k_{T}$ algorithm [56]. The jets that satisfy $p_{T}^{\text {jet }}>30 \mathrm{GeV}$ and $|\eta|<4.5$ are retained for further analysis.

(6) For the topologies which require $b$ tagging, $\Delta R$ is computed between a $b$ parton and each of the anti- $k_{T}$ jets. If it happens to be less than 0.1 , we convolute it with an additional $70 \% b$-tag efficiency factor.

Note that the above cuts (criteria) imposed on the finalstate objects in PYTHIA are extremely generic and not specific to any process under consideration. Hence these fall under the category of preselection cuts. In the coming sections, we discuss the detailed collider analysis of observing the light Higgs boson. The signal significance ${ }^{7}$ is computed over the allowed parameter space as a function of $\alpha, \tan \beta$, and $m_{h}$.

\section{A. Channel 1: $p p \rightarrow h \rightarrow \gamma \gamma$}

We begin with the analysis of the light Higgs boson decaying to the diphoton final state. For our signal topology, the irreducible background arises from the tree-level quark-antiquark as well as loop-induced gluon-gluon annihilation to $\gamma \gamma$. The reducible backgrounds arise from $j \gamma, j j$, and $e^{+} e^{-}$final states, respectively, where a jet(s) or lepton (s) fakes a photon(s). The QCD backgrounds can be considerably reduced by demanding the final-state photons to be isolated (see Table III). The background due to the $Z$-pole contributions in the Drell-Yan $(Z \rightarrow e e)$ process also dilutes the diphoton signal for light Higgs masses around $m_{Z}$ due to its large cross section, even though the mistagging rate for an electron to fake a photon is small.

\footnotetext{
${ }^{7}$ The significance $S$ of observing signal over background is defined as $\frac{s}{\sqrt{s+b}}$, where $s$ and $b$ are the number of signal and background events, respectively.
}

TABLE III. The efficiencies of the signal and background processes against different cuts are listed for Channel 1. The light Higgs mass is chosen to be $110 \mathrm{GeV}$ for illustration. The dijet background becomes negligible after imposing all of the cuts.

\begin{tabular}{lccccc}
\hline \hline & \multicolumn{5}{c}{ Efficiency } \\
\cline { 2 - 6 } & & \multicolumn{4}{c}{ Backgrounds } \\
\cline { 2 - 6 } Cuts & Signal & $\gamma \gamma$ & $j \gamma$ & $j j$ & $e e$ \\
\hline Preselection & 0.59 & 0.377 & 0.019 & $1.0 \times 10^{-6}$ & $1.0 \times 10^{-3}$ \\
$p_{T}$ selection & 0.84 & 0.28 & 0.21 & $\sim 0$ & 0.45 \\
$m_{\text {inv }}^{\gamma \gamma}$ selection & 0.99 & 0.082 & 0.024 & 0 & $\sim 10^{-4}$ \\
\hline \hline
\end{tabular}

The preselection criteria discussed in the previous section are extremely generic and cannot aid in effective signalbackground separation. Additional cuts on the kinematic variables [i.e., the transverse momentum $\left(p_{T}\right)$ and the invariant mass of the diphoton pair] are necessary for further reduction in the background processes. To illustrate this point, in Fig. 6 we plot the normalized transverse momentum distributions for the leading isolated photon $\left(p_{T}^{\gamma}\right)$ corresponding to the signal (with $m_{h}=110 \mathrm{GeV}$ ) and the SM backgrounds. The $p_{T}$ for the signal distribution peaks approximately at $m_{h} / 2$, and for backgrounds processes (e.g., $\gamma \gamma, j \gamma$, and $j j$ ) it peaks at much lower values (although in the plot only the $\gamma \gamma$ background is shown). Therefore, a suitable choice of the cuts on the leading and subleading isolated photon candidates and the invariant mass of the diphoton pair can enhance the signal significance. The selection cuts used for the diphoton analysis are as follows:

$$
\begin{aligned}
& p_{T} \text { selection: } p_{T_{\text {lead }}}^{\gamma}>40 \mathrm{GeV}, p_{T_{\text {sub }}}^{\gamma}>30 \mathrm{GeV}, \\
& m_{\text {inv }}^{\gamma \gamma} \text { selection: }\left|m_{\text {inv }}^{\gamma \gamma}-m_{h}\right|<2.5 \mathrm{GeV} .
\end{aligned}
$$

Here $p_{T_{\text {lead }}}^{\gamma}$ and $p_{T_{\text {sub }}}^{\gamma}$ correspond to the transverse momentum of the leading and subleading photon, respectively, and $m_{\text {inv }}^{\gamma \gamma}$ corresponds to the invariant mass of the diphoton pair. Table III shows the efficiencies of the preselection and selection cuts on the signal and background processes, where the efficiency of a cut is defined as

Efficiency $\equiv \frac{\text { Number of events after imposing the cut }}{\text { Number of events before imposing the cut }}$.

After imposing the preselection and selection cuts on the signal and background processes, we are in a position to determine the signal significance for the light Higgs boson as a function of its mass and mixing angles $\alpha$ and $\beta$. In Fig. 7(a) we plot the significance of observing $h$, i.e., $S(\gamma \gamma)$ with respect to $\alpha$ for $m_{h}=100 \mathrm{GeV}$ and an integrated luminosity $\mathcal{L}=300 \mathrm{fb}^{-1}$ for different values of $\tan \beta$. In Fig. 7(b) we repeat the exercise with $m_{h}=110 \mathrm{GeV}$. Note that the significance for smaller masses is negligible, and hence it is not shown in the plot. We now discuss the 


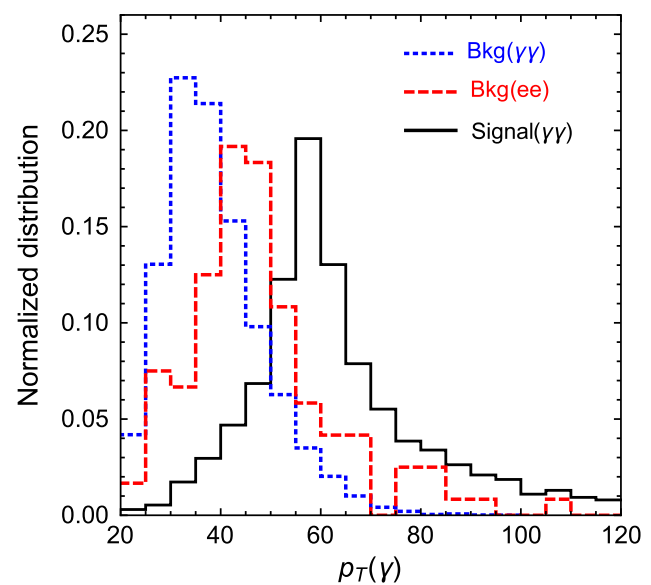

FIG. 6. The figure illustrates the normalized $p_{T}$ distributions of the leading isolated photon in the channel $\gamma \gamma$ for the signal and background processes. Here $\operatorname{Signal}(\gamma \gamma)$ corresponds to the light Higgs boson of mass $m_{h}=110 \mathrm{GeV}$, which is produced in the gluon-fusion process and decays to a pair of photons, $\operatorname{Bkg}(\gamma \gamma)$ corresponds to the irreducible diphoton background, and $\operatorname{Bkg}(e e)$ corresponds to reducible background where both electrons fake a photon.

qualitative features of Fig. 7 with respect to $\alpha$ and $\tan \beta$. The discontinuities in Fig. 7(a) for $\tan \beta=3$ and 4 near the fermiophobic limit correspond to the excluded regions from the direct searches of the light Higgs at the LHC, as discussed in Sec. IV B. In addition, the constraints from LEP limit the allowed range of $\alpha$ to be less than $\pi$ for a given light Higgs mass and $\tan \beta$ (see discussions in Sec. IV B). The dip in the significance signifies the regions where the total cross section proportional to $\xi_{h}^{f} \times \xi_{h}^{\gamma}$ vanishes. The first minimum occurs where $\xi_{h}^{\gamma}$ vanishes due to cancellation of the top and $W$ loop contribution ${ }^{8}$ in $h \rightarrow \gamma \gamma$ whereas the second minimum corresponds to the fermiophobic limit $\left(\xi_{h}^{f} \rightarrow 0\right)$. Hence, this channel is ineffective in probing the regions close to the fermiophobic limit. The significance of observing the signal in this channel is larger for $\alpha>\pi / 2$ as $\sin (\beta-\alpha)$ is negative in this region. As a consequence, the top- and $W$-loop interfere constructively and enhance the diphoton rate.

\section{B. Channel 2: $p p \rightarrow W h \rightarrow W \gamma \gamma$}

In this section, we analyze the discovery prospects of the light Higgs boson in the channel $W \gamma \gamma$ at $13 \mathrm{TeV}$ center-ofmass energy, where the leptonic decays (only $e$ and $\mu$ ) of $W$ are considered. The SM backgrounds arises from $p p \rightarrow W \gamma \gamma, \quad p p \rightarrow W j \gamma, \quad p p \rightarrow W j j, \quad$ and $p p \rightarrow W Z$ $\left(Z \rightarrow e^{+} e^{-}\right)$. The background reduction methods are exactly the same as the ones discussed in Sec. VA, and hence we refrain from discussing them in this section.

\footnotetext{
${ }^{8}$ For large values of $\tan \beta$, the dip corresponding to $\xi_{h}^{\gamma} \rightarrow 0$ shifts towards $\alpha \approx \pi / 2$ [see Eq. (A3)].
}

The signal is characterized by the presence of at least one isolated lepton, two isolated photons, and missing energy. The selection cuts used in the analysis are

$$
\begin{array}{cc}
p_{T} \text { selection: } & p_{T}^{\ell}>30 \mathrm{GeV}, E_{T}^{\text {miss }}>30 \mathrm{GeV}, \\
& p_{T_{\text {lead }}}^{\gamma}>40 \mathrm{GeV}, p_{T_{\text {sub }}}^{\gamma}>30 \mathrm{GeV}, \\
m_{\text {inv }}^{\gamma \gamma} \text { selection: } & \left|m_{\text {inv }}-m_{h}\right|<2.5 \mathrm{GeV} .
\end{array}
$$

Here $p_{T}^{\ell}$ corresponds to the transverse momentum of leptons $(e$ and $\mu)$ and $E_{T}^{\text {miss }}$ denotes the total missing transverse energy. We refer to Table IV for the effect of preselection and selection cuts on the signal and background processes. This channel allows us to probe the regions close to fermiophobic limit where production via the gluon-fusion process loses its sensitivity. In Fig. 8, the significance $S(\ell \nu \gamma \gamma)$ of the signal with respect to $\alpha$ for $100 \mathrm{fb}^{-1}$ integrated luminosity is plotted for four different values of the mass of the light Higgs. We now summarize the distinctive features of Fig. 8 below:

(1) For a given light Higgs mass, the significance in this channel decreases with as $\tan \beta$ increases, as the production cross section (proportional to $\xi_{h}^{V}$ ) decreases for large values of $\tan \beta$.

(2) The branching ratio of $h \rightarrow W W^{*}$ increases significantly for larger values of $m_{h}$. Furthermore, the decay $h \rightarrow Z \gamma$ also opens up for $m_{h}>m_{Z}$. As a result, the branching ratio of the light Higgs to diphotons decreases with as $m_{h}$ increases. This reduces the signal significance substantially.

(3) The discontinuities in Fig. 8 correspond to the disallowed regions from the LEP and LHC direct search measurements. The chopped-off upper half of the curves in Fig. 8(a) for $\tan \beta=4,6$, Fig. 8(b) for $\tan \beta=3,4,6$, and Fig. 8(c) for $\tan \beta=3,4$ near $\alpha \approx \pi / 2$ are due to the LHC constraint. These are exactly the disallowed wedge-shaped regions in Fig. 5. Note that the bounds from the LHC become insignificant for larger values of $\tan \beta$ and $m_{h}$. This can be easily seen in Fig. 5. The discontinuites are absent in Fig. 8(a) for $\tan \beta=10$, Fig. 8(b) for $\tan \beta=10$, Fig. 8(c) for $\tan \beta=6,10$ and Fig. 8(d) for $\tan \beta=3,4,6,10$. The direct search bounds from LEP, on the other hand, constrain the minimum and maximum values of $\alpha$. This restricts the net allowed range of $\alpha$ to be less than $\pi$. Since in Fig. 8 we highlight regions close to the fermiophobic limit, the net effect of the LEP constraints is not visible.

To conclude, regions around the fermiophobic limit can be best explored at the $13 \mathrm{TeV}$ LHC for lower masses of the light Higgs and intermediate $\tan \beta$ values.

\section{Channel 3: $p p \rightarrow W h \rightarrow W b \bar{b}$}

In this section, we analyze the discovery prospects of the light Higgs in the $W b \bar{b}$ channel, where we consider leptonic decays of $W$. The signal is characterized by 

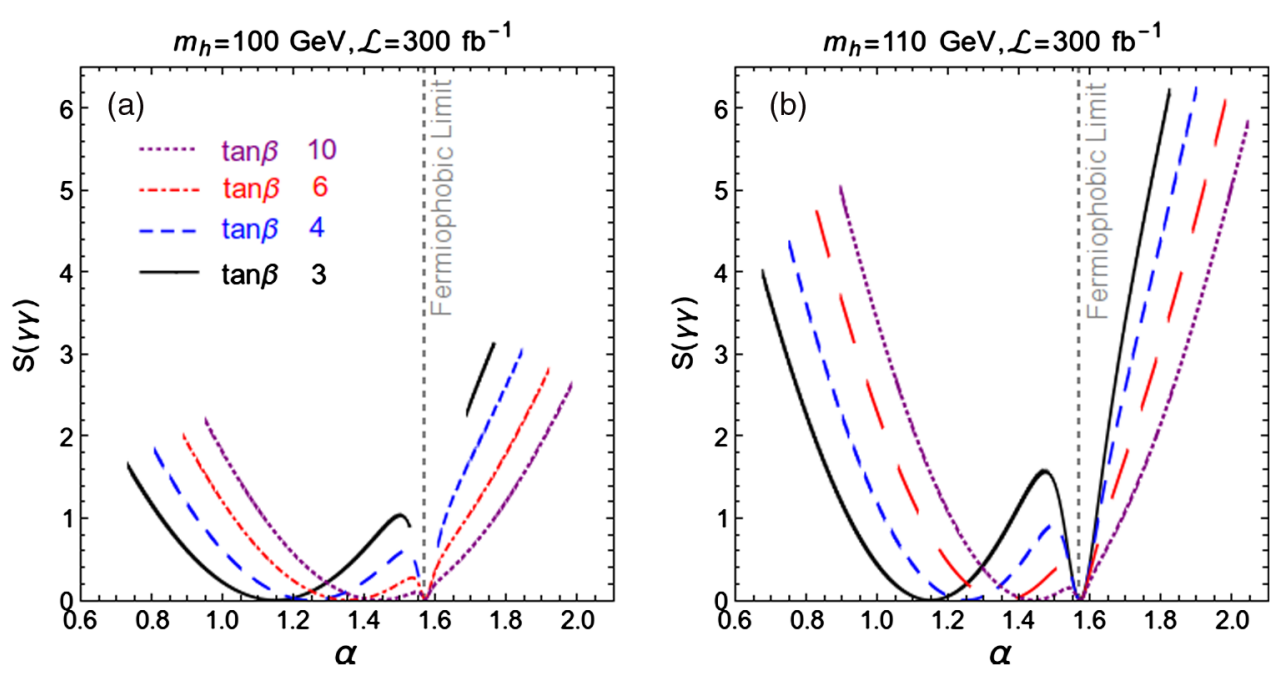

FIG. 7. Variation of the signal significance $S(\gamma \gamma)$ with $\alpha$ for $m_{h}=100,110 \mathrm{GeV}$ and $\mathcal{L}=300 \mathrm{fb}^{-1}$ for different values of tan $\beta$. Panel (a) corresponds to $m_{h}=100 \mathrm{GeV}$ and panel (b) to $110 \mathrm{GeV}$. The vertical gray dashed line corresponds to $\alpha=\pi / 2$, i.e., the fermiophobic limit. Here the signal significance drops to zero, as expected. Hence, the light Higgs produced in gluon fusion is insensitive to the alignment limit. The discontinuities in panel (a) for $\tan \beta=3$ and 4 near the fermiophobic limit correspond to the excluded regions from the direct searches for the light Higgs at the LHC, as discussed in Sec. IV B.

$W b \bar{b}$, where we tag the leptonic $(e$ and $\mu$ ) decays of $W$. The signal is categorized by the presence of two $b$-tagged jets, an isolated lepton, and missing energy. In spite of the fact that it is the dominant decay channel in most of the parameter space, the $b \bar{b}$ mode is difficult to probe because of the presence of the enormous QCD background. The SM irreducible background arises from $p p \rightarrow W Z$. The reducible background arise from $p p \rightarrow t \bar{t}$ where one of the $W$ 's is along the beam line and hence escapes detection, and $W+$ jets where light-quark jets are mistagged as $b$-jets. The $W h$ production rate is governed by the magnitude of $\xi_{h}^{V}$ and is small in the favored parts of the parameter space. With a small signal cross section in comparison to large backgrounds, it is difficult to isolate signal events from huge SM backgrounds in the $2 b+\ell+E_{T}^{\text {miss }}$ final state at the LHC. In order to achieve appreciable significance at the LHC, we follow the analysis of Ref. [36] and consider the $W h$ process in the boosted regime. Although we lose a significant number of events by demanding a boosted Higgs $\left(p_{T}^{h}>200 \mathrm{GeV}\right)$, it enables us to overcome the huge SM backgrounds quite efficiently. We reconstruct a fat jet

TABLE IV. The efficiencies of the signal and background processes against different cuts for Channel 2. The light Higgs mass is chosen to be $110 \mathrm{GeV}$ for illustration.

\begin{tabular}{lcccc}
\hline \hline & \multicolumn{4}{c}{ Efficiency } \\
\cline { 2 - 5 } & & \multicolumn{3}{c}{ Backgrounds } \\
\cline { 2 - 5 } Cuts & Signal & $W \gamma \gamma$ & $W j \gamma$ & Wee \\
\hline Preselection & 0.29 & 0.042 & 0.032 & $4.9 \times 10^{-3}$ \\
$p_{T}$ selection & 0.55 & 0.186 & 0.36 & 0.308 \\
$m_{\text {inv }}^{\gamma \gamma}$ selection & 0.98 & 0.028 & 0.023 & $6 \times 10^{-3}$ \\
\hline \hline
\end{tabular}

with radius parameter $R_{J}=0.8$ and transverse momentum $p_{T}^{J}>200 \mathrm{GeV}$. We then tag the fat jet as a Higgs using the mass-drop technique discussed in Appendix C.

The analysis is performed with $14 \mathrm{TeV}$ center-of-mass energy for $m_{h}=70,80,100$ and $110 \mathrm{GeV}$. We have not considered $m_{h}=90 \mathrm{GeV}$ in our analysis as it is difficult to isolate the signal from the huge $Z \rightarrow b \bar{b}$ background. We summarize our selection criteria as follows:

$p_{T}^{\ell}>30 \mathrm{GeV}, \quad E_{T}^{\text {miss }}>30 \mathrm{GeV}$,

$p_{T}^{W}=\left|p_{T}^{\ell}+\mathbf{p}_{\mathbf{T}}^{\text {miss }}\right|>200 \mathrm{GeV}, \quad R_{J}=0.8$,

$p_{T}^{J}>200 \mathrm{GeV}, \quad\left|m_{h}-m_{J}\right|<5 \mathrm{GeV} \quad\left(\right.$ for $\left.m_{h} \leq 90 \mathrm{GeV}\right)$,

$p_{T}^{J}>250 \mathrm{GeV}, \quad\left|m_{h}-m_{J}\right|<8 \mathrm{GeV} \quad\left(\right.$ for $\left.m_{h}>90 \mathrm{GeV}\right)$,

where $p_{T}^{W}$ is the magnitude of the vector sum of the momentum of the lepton and missing energy in the transverse plane. The efficiencies of these cuts are displayed in Table V. We can see that by demanding at least one fat jet and anti- $k_{T}$ jet reduces the $W b \bar{b}$ and $W 3 j$ backgrounds. Also, by invoking a fat jet with no jet activity outside and MassDrop with a double $b$-tag, we are able to suppress the $t \bar{t} b \bar{b}$ process very effectively.

After imposing the above cuts, we compute the signal significance for the light Higgs boson as a function of its mass and mixing angles $\alpha$ and $\beta$. In Fig. 9 we plot the significance of observing the light Higgs as a function of $\alpha$. Figures 9(a) and 9(b) represent the significance with an integrated luminosity of $300 \mathrm{fb}^{-1}$ for $m_{h}=100$ and $110 \mathrm{GeV}$, respectively. Again, the discontinuities in Fig. 9 arise due to direct detection constraints from LEP and LHC. It is interesting to note the behavior of the signal significance in Fig. 9. The dip in the plot signifies the points 

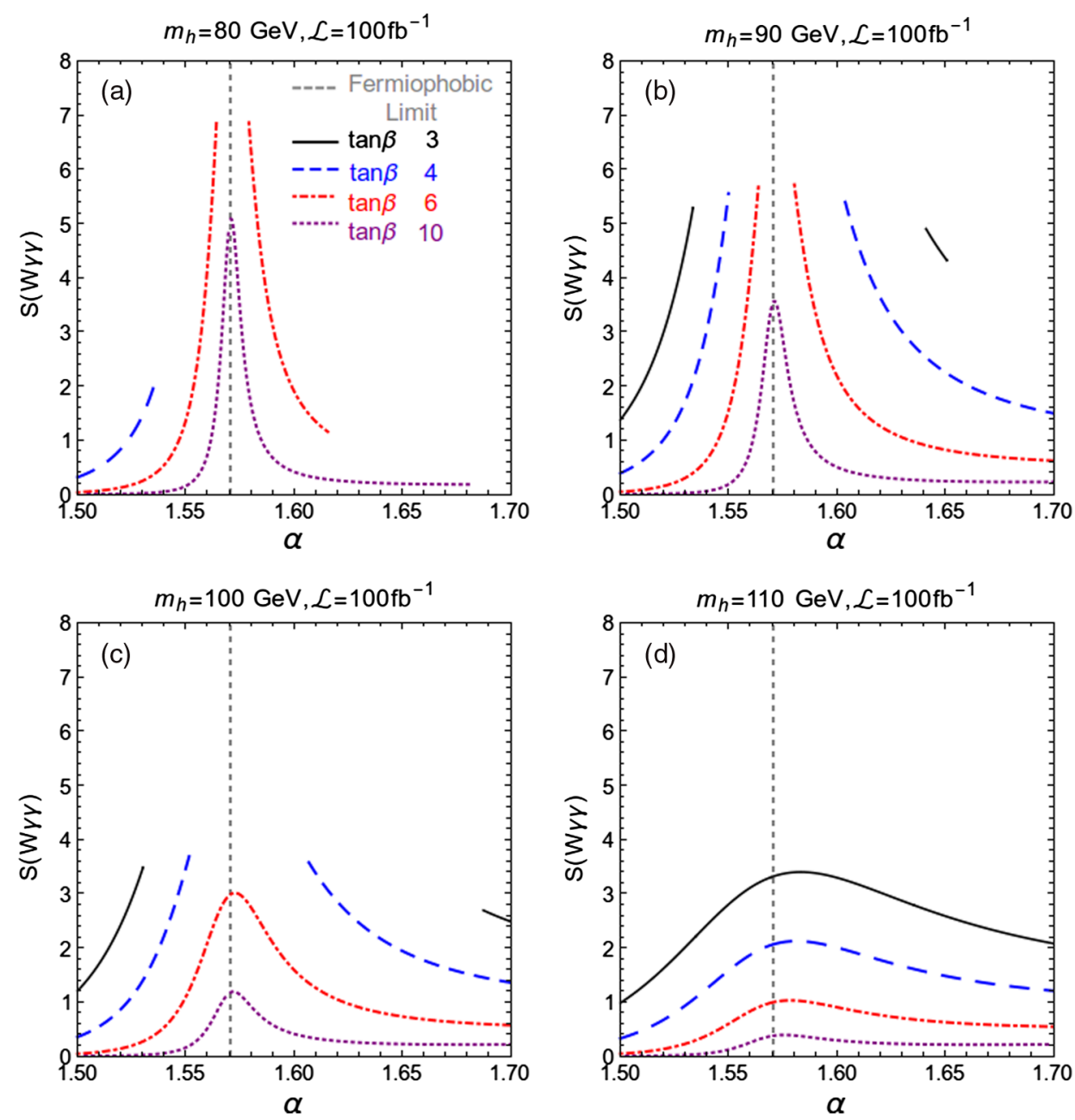

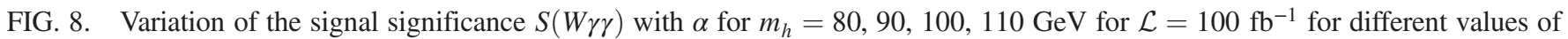
$\tan \beta$. The color code is the same as in Fig. 7. Note that the range of $\alpha$ is restricted in the plot to signify the regions with reasonable significance. The discontinuities in panel (a) for $\tan \beta=4$ and 6 , in panel (b) for $\tan \beta=3,4$ and 6 , and in panel (c) for $\tan \beta=3$ and 4 near the fermiophobic limit correspond to the excluded regions from the direct searches of the light Higgs at LHC-I, as discussed in Sec. IV B. Note that the bounds from the LHC-I become insignicant for larger values $m_{h}$, that is why there are no discontinuities present in panel (d). The absence of the $\tan \beta=3$ line in panel (a) is attributed to constraints from LEP which set an upper limit on $\alpha$ and require it to be less than 1.5 for $\tan \beta=3$ and $m_{h}=80 \mathrm{GeV}$.

TABLE V. The efficiencies of the different cuts used for the analysis of Channel 3 for both signal and background processes. The numbers are for a light Higgs mass of $110 \mathrm{GeV}$.

\begin{tabular}{lllll}
\hline \hline & \multicolumn{4}{c}{ Efficiency } \\
\cline { 2 - 5 } & & \multicolumn{3}{c}{ Backgrounds } \\
\cline { 2 - 5 } & Signal & $W b b$ & $W 3 j$ & $t \bar{t} b \bar{b}$ \\
\hline Cuts & 0.45 & 0.11 & 0.10 & 0.47 \\
$\quad$ At least one fat jet and anti- $k_{T}$ & & & & \\
$\quad$ jet & 0.86 & 0.71 & 0.68 & 0.21 \\
Isolated leptons & 0.5 & 0.27 & 0.16 & 0.019 \\
One fat jet with no anti- $k_{T}$ jet & 0.987 & 0.93 & 0.93 & 0.99 \\
$E_{T}^{\text {miss }}>30 \mathrm{GeV}$ & 0.93 & 0.88 & 0.85 & 0.77 \\
$p_{T}^{W}>200 \mathrm{GeV}$ & 0.32 & 0.299 & 0.0037 & 0.031 \\
MassDrop with double $b$-tag & 0.79 & 0.077 & 0.077 & 0.11 \\
Invariant mass & & &
\end{tabular}

where the total cross section proportional to $\xi_{h}^{V} \times \xi_{h}^{f}$ vanishes. The first dip corresponds to $\xi_{h}^{V} \rightarrow 0$ and the second dip represents $\xi_{h}^{f} \rightarrow 0$ (fermiophobic limit). Hence, this channel is useful in probing regions away from the fermiophobic limit.

\section{Channel 4: $p p \rightarrow t \bar{t} h \rightarrow t \bar{t} b \bar{b}$}

Continuing with the discussion of a light Higgs decaying to $b \bar{b}$, we now focus our attention on the $t \bar{t} h$ production mode, where semileptonic decays of top pairs are considered. The irreducible background here arises from the $t \bar{t} b \bar{b}$ final state and the reducible background arises from $t \bar{t}+$ jets, where a jet fakes the bottom quark. Due to the presence of four $b$ quarks in the final state, it is difficult to reconstruct the light Higgs accurately due to the various possible combinations. This problem can be addressed by resorting to boosted 

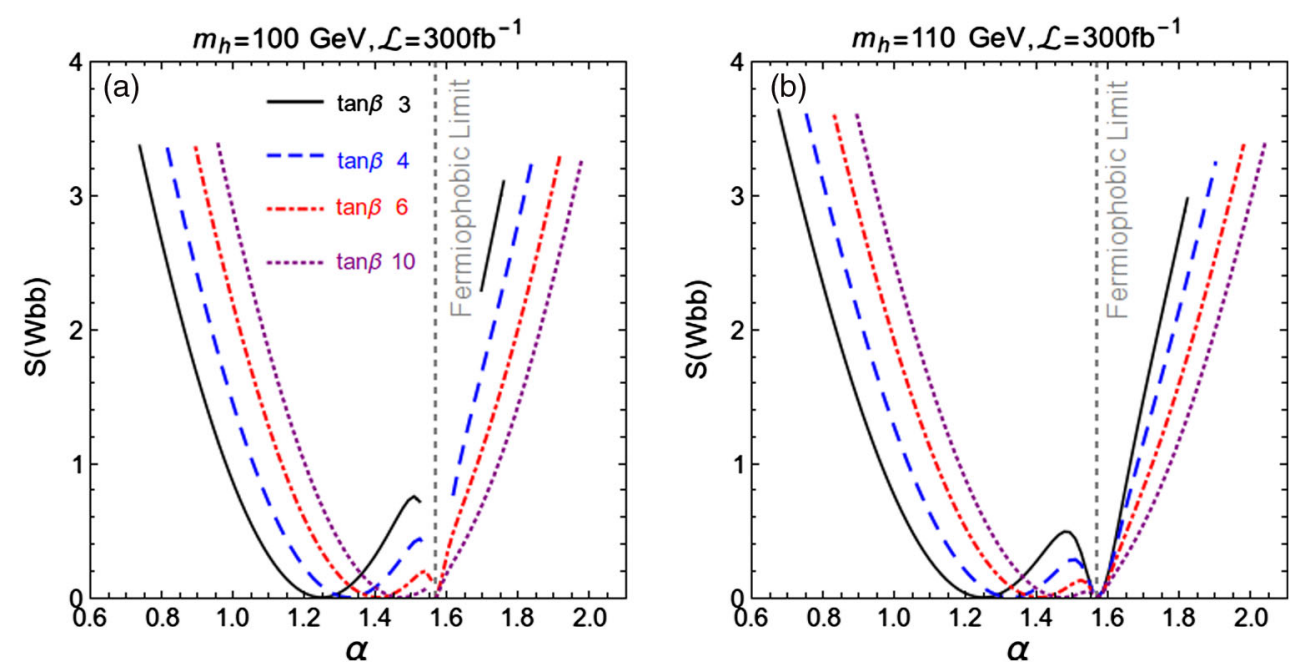

FIG. 9. Variation of the signal significance $S(W b b)$ with $\alpha$ for $m_{h}=100 \mathrm{GeV}$ and $110 \mathrm{GeV}$ for $\mathcal{L}=300 \mathrm{fb}^{-1}$ for different values of $\tan \beta$ are illustrated. Panel (a) corresponds to $m_{h}=100 \mathrm{GeV}$ and panel (b) to $110 \mathrm{GeV}$. The color code is the same as that in Figs. 7 and 8. This channel is also insensitive around the fermiophobic limit.

scenarios where the decay products of the hadronically decaying top and light Higgs are enclosed within a single jet of large radius parameter. Therefore, our signal essentially comprises of two fat jets, an isolated lepton, missing energy, and one anti- $k_{T} b$-tagged jet. To tag the top and Higgs jets, we first construct the fat jets with $p_{T}^{J}>125 \mathrm{GeV}$ and $\Delta R=1.2$. The jets satisfying $p_{T}^{J}>250 \mathrm{GeV}$ are tagged as top jets if they satisfy the prescription described in Appendix C.
Similarly, the remaining jets are tagged as the Higgs jets if they satisfy the mass-drop criteria and the filtered jet mass, $m_{J}^{\text {Higgs }}$, lies within a $5 / 10 \mathrm{GeV}$ window about the light Higgs mass (see Appendix $\mathrm{C}$ for more details). In addition, we demand a $b$-tagged jet outside the top and Higgs fat jet. This helps in further eliminating the $t \bar{t}+$ jets background. We summarize the cuts used in the analysis below:

$$
\begin{aligned}
p_{T}^{\ell} & >30 \mathrm{GeV}, & E_{T}^{\text {miss }}>30 \mathrm{GeV}, \quad p_{T}^{\text {top }}>250 \mathrm{GeV}, \quad 150 \mathrm{GeV}<m_{J}^{\text {top }}<200 \mathrm{GeV}, \\
p_{T}^{\text {Higgs }}>125 \mathrm{GeV}, & & \left|m_{J}^{\text {Higgs }}-m_{h}\right|<5 \mathrm{GeV} \quad\left(\text { for } m_{h} \leq 90 \mathrm{GeV}\right), \\
p_{T}^{\text {Higgs }}>160 \mathrm{GeV}, & & \left|m_{J}^{\text {Higgs }}-m_{h}\right|<10 \mathrm{GeV} \quad\left(\text { for } m_{h}>90 \mathrm{GeV}\right) .
\end{aligned}
$$

The efficiencies of the individual cuts are listed in Table VI. We are now in a position to estimate the signal significance, i.e., $S(\bar{t} \bar{t} b \bar{b})$ as a function of $\alpha, \tan \beta$, and $m_{h}$. In Fig. 10 we plot the significance of observing a light Higgs for four different light Higgs masses: $m_{h}=70,80,100$, and $110 \mathrm{GeV}$. We have not considered $m_{h}=90 \mathrm{GeV}$ for the analysis because in that case it will be difficult to isolate the signal events from the large $t \bar{t} Z$ background. Note that we have chosen smaller $\tan \beta$ values as the total cross section decreases with as $\beta$ increases (see Table VII). The significance is higher for lower values of $\alpha$. Hence this channel is effective for probing lower $\tan \beta$ and $\alpha$ regions. This particular mode for probing the light Higgs does not work out in the fermiophobic limit as both the production cross section and decay branching ratio are negligible.

\section{E. Summary and concluding remarks}

To summarize, we studied the prospects of observing a $C P$-even scalar lighter than the observed $125 \mathrm{GeV}$ Higgs at the LHC, in the context of the type-I 2HDM. We identified the heavier $C P$-even Higgs in the 2HDM with the discovered Higgs. We also considered the charged and

TABLE VI. The efficiencies of the different cuts used for the analysis of Channel 4 for both signal and background processes. The numbers are for a light Higgs mass of $110 \mathrm{GeV}$.

\begin{tabular}{llll}
\hline \hline & \multicolumn{3}{c}{ Efficiency } \\
\cline { 2 - 4 } & & \multicolumn{2}{c}{ Backgrounds } \\
\cline { 2 - 4 } Cuts & Signal & $t t b b$ & $t t+3 j$ \\
\hline Isolated leptons & 0.53 & 0.56 & 0.57 \\
Two fat jets & 0.31 & 0.17 & 0.20 \\
$p_{T}^{\ell}>30 \mathrm{GeV}, E_{T}^{\text {miss }}>30 \mathrm{GeV}$ & 0.76 & 0.65 & 0.63 \\
$\quad$ Top tagged & 0.11 & 0.088 & 0.13 \\
$\quad \begin{array}{l}\text { Mass drop with double } b \text {-tag } \\
\quad \text { and invariant mass }\end{array}$ & 0.056 & 0.011 & 0.0009 \\
$\quad \begin{array}{llll}\text { Anti- } k_{T} b \text {-jet outside top } \\
\quad \text { and Higgs jet }\end{array}$ & 0.28 & 0.25 & 0.50 \\
\hline \hline
\end{tabular}



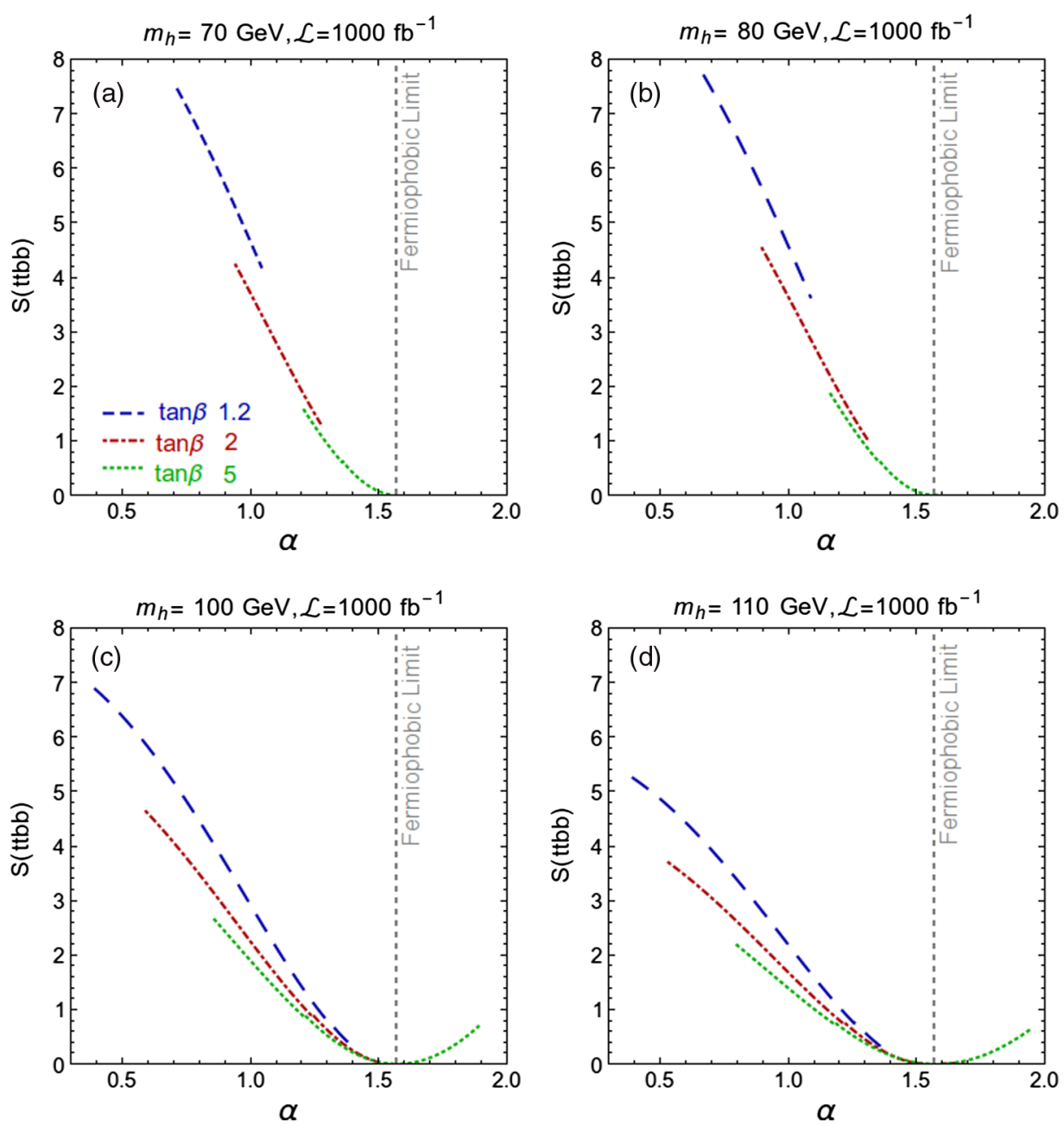

FIG. 10. Variation of signal significance $S(\bar{t} \bar{t} b \bar{b})$ in the channel $t \bar{t} b \bar{b}$ with $\alpha$ for different values of $m_{h}=70$ (a), 80 (b), 100 (c) and $110 \mathrm{GeV}(\mathrm{d})$ at $1000 \mathrm{fb}^{-1}$ integrated luminosity. While the dark blue, dark red, and dark green dashed lines correspond to tan $\beta=1.2,2$, and 5, respectively, the dashed gray vertical line for $\alpha=\pi / 2$ illustrates the fermiophobic limit. Owing to the enhanced sensitivity of $\sigma(p p \rightarrow t \bar{t} h \rightarrow t \bar{t} b \bar{b})$ for low $\tan \beta$, we have chosen slightly lower values of $\tan \beta$ for this channel.

pseudoscalar Higgs bosons to be heavy. This choice simplifies the $2 \mathrm{HDM}$ parameter space and leaves $\alpha$, $\tan \beta$, and the mass of the light Higgs $\left(m_{h}\right)$ as the relevant free parameters. We considered various theoretical and experimental constraints to determine the allowed regions in the parameter space. The mass of the light Higgs was taken to be greater than $62.5 \mathrm{GeV}$ to avoid $H \rightarrow h$ h decay.

To study the phenomenology of the light Higgs at the LHC, we determined the suitable production and decay modes. In most parts of the parameter space, the light Higgs in the type-I $2 \mathrm{HDM}$ decays dominantly to $b \bar{b}$. However, for regions close to the fermiophobic limit, its decay to bosons (mainly photons) becomes dominant. Therefore, we focused on the light Higgs decay to $b \bar{b}$ and $\gamma \gamma$ in this analysis. Analyzing $b \bar{b}$ in the $g g F$ or VBF production mode is challenging due to the large QCD background. We chose the light Higgs production in association with the $W$ boson and top pair for the $b \bar{b}$ analysis. Furthermore, we tagged the light Higgs in the boosted regimes for better signal significances. The choice of the production mode for the $\gamma \gamma$ channel is much simpler because of its better reconstruction properties. We chose the $W h$ production mode to analyze regions close to the fermiophobic limit, and the $g g F$ production mode for regions away from the fermiophobic limit.

We analyzed the discovery prospects of the light Higgs boson in four channels at the LHC: $p p \rightarrow h \rightarrow \gamma \gamma$, $p p \rightarrow W h \rightarrow W \gamma \gamma, p p \rightarrow W h \rightarrow W b \bar{b}$, and $p p \rightarrow t \bar{t} h \rightarrow$ $t \bar{t} b \bar{b}$. We found interesting regions in the parameter space of the $2 \mathrm{HDM}$ that could be probed at the future runs of the LHC with a few hundred $\mathrm{fb}^{-1}$ of luminosity. We summarize our findings in Fig. 11 for $m_{h}=110 \mathrm{GeV}$ and $\mathcal{L}=300 \mathrm{fb}^{-1}$. In this plot, the yellow contour illustrates the total allowed region for $m_{h}=110 \mathrm{GeV}$. The hatched portions denote the regions where the above channels could be probed with significances greater than 


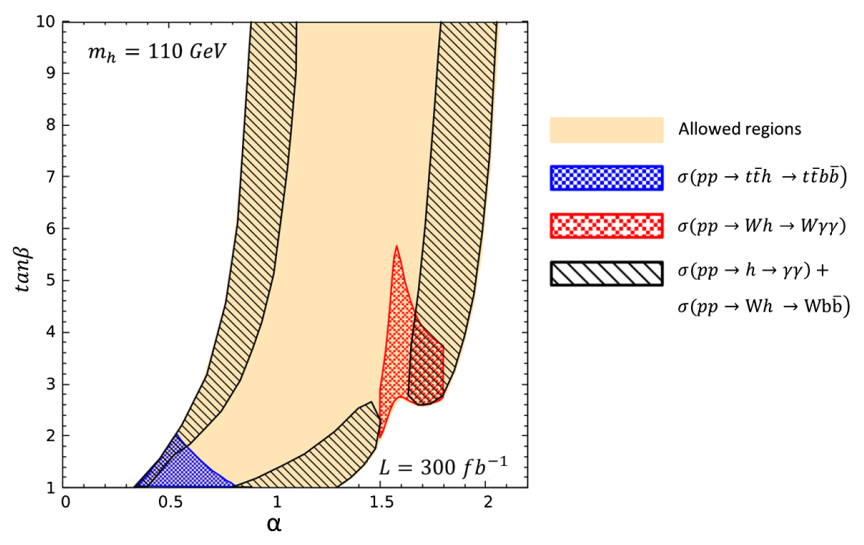

FIG. 11. Regions of the allowed parameter space that could be probed/excluded in different channels with significances greater than $2 \sigma$ for a light Higgs boson of mass $110 \mathrm{GeV}$ at the LHC with $300 \mathrm{fb}^{-1}$ of integrated luminosity. While the yellow contour illustrates the total allowed region in the $(\alpha, \tan \beta)$ plane for $m_{h}=110 \mathrm{GeV}$, we can probe only the hatched regions with significances greater than $2 \sigma$, leaving behind regions which satisfy the alignment limit $\alpha \approx \beta$. Here we have combined the allowed regions for $p p \rightarrow h \rightarrow \gamma \gamma$ and $p p \rightarrow W h \rightarrow W b \bar{b}$ due to their similar behavior.

$2 \sigma$ at the LHC. The unhatched regions in the allowed contour correspond to $\alpha \approx \beta$ and approximately satisfy the alignment limit. As already noted, such regions would be difficult to probe/rule out in the near future. For the purpose of the plot, we have combined the allowed regions for $p p \rightarrow h+X \rightarrow \gamma \gamma+X$ and $p p \rightarrow W h+X \rightarrow W b \bar{b}+X$ as they probe almost similar parts of the parameter space (see Appendix D).

Searches for physics beyond the Standard Model to date have yielded neither any significant results nor specific directions to follow. However, the current measurements still do not rule out the possibility of the observed $125 \mathrm{GeV}$ scalar belonging to some enlarged sector. In this paper, we examined a possible scenario in the context of the type-I 2HDM and studied the prospects of observing a light $C P$-even scalar at the future runs of the LHC. Our aim in this study was to put together all of the relevant information and provide an optimized search strategy for the light Higgs at the LHC. The discovery of such a light scalar would not only open doors to new physics, but also help us to better understand the mechanism of electroweak symmetry breaking.

\section{ACKNOWLEDGMENTS}

We would like to thank Sandhya Jain, Manoranjan Guchait, and Tuhin Roy for helpful discussions. We would also like to acknowledge Amol Dighe, Debjyoti Bardhan, Sreerup Raychaudhuri, and Tuhin Roy for careful reading of our manuscript. S. N. acknowledges the Dr. D. S. Kothari Post Doctoral Fellowship awarded by University Grant
Commission (Award Letter No. PH/15-16/0073) for financial support. We would also like to thank the Department of Theoretical Physics, TIFR for the use of its computational resources.

Note added.-Recently, the CMS Collaboration reported a local excess of $2.8 \sigma$ (global $1.3 \sigma$ ) in the $\gamma \gamma$ channel around $95 \mathrm{GeV}$ [51]. Although at this stage the deviation is not significant, it is certainly of importance simply because LEP had also indicated a possibility of observing an excess of Higgs-like events in a similar mass region [57]. Therefore, it is important to analyze theories that predict a low-mass scalar and that can explain such an excess. The type-I $2 \mathrm{HDM}$, in this context, perfectly fits the bill. Since the excess at present is only indicative, we have restricted ourselves to a generic low-mass analysis in the type-I 2HDM. In particular, for implications of the type-I 2HDM in the light of the recent CMS result, one could refer to Refs. [58,59].

\section{APPENDIX A: DIPHOTON LOOP}

The effective interactions of $h(H)$ with $\gamma \gamma$ are given as [60]

$$
L=\frac{\alpha_{e m}}{8 \pi v} \xi_{h}^{\gamma} h F_{\mu \nu} F^{\mu \nu}+\frac{\alpha_{e m}}{8 \pi v} \xi_{H}^{\gamma} H F_{\mu \nu} F^{\mu \nu}
$$

Correspondingly, the decay width is

$$
\Gamma(h(H) \rightarrow \gamma \gamma)=\frac{\alpha^{2} g^{2}}{1024 \pi^{3}} \frac{m_{h(H)}^{3}}{m_{W}^{2}}\left|\xi_{h(H)}^{\gamma}\right|^{2} .
$$

For the type-I 2HDM, the effective couplings $\xi_{h(H)}^{\gamma}$ receive dominant contributions from tje $W$ boson, charged Higgs, and top loop, and are given as

$$
\begin{aligned}
\xi_{h(H)}^{\gamma}= & N_{c} Q_{t}^{2} \xi_{h(H)}^{t} F_{1 / 2}\left(\tau_{t}\right)+\xi_{h(H)}^{W} F_{1}\left(\tau_{W}\right) \\
& +\frac{m_{W}^{2}}{M_{H^{ \pm}}^{2}} \xi_{h(H)}^{H^{ \pm}} F_{0}\left(\tau_{H^{ \pm}}\right) .
\end{aligned}
$$

The form factors are given as

$$
\begin{aligned}
& F_{0}\left(\tau_{H^{ \pm}}\right)=\tau_{H^{ \pm}}\left[1-\tau_{H^{ \pm}} f\left(\tau_{H^{ \pm}}\right)\right] \\
& F_{1 / 2}\left(\tau_{t}\right)=-2 \tau_{t}\left[1+\left(1-\tau_{t}\right) f\left(\tau_{t}\right)\right], \\
& F_{1}\left(\tau_{W}\right)=2+3 \tau_{W}+3 \tau_{W}\left(2-\tau_{W}\right) f\left(\tau_{W}\right),
\end{aligned}
$$

where 


$$
\begin{aligned}
f(\tau) & =\left(\sin ^{-1} \frac{1}{\sqrt{\tau}}\right)^{2} \quad \text { for } \tau>1, \quad f(\tau)=-\frac{1}{4}\left(\log \frac{\eta_{+}}{\eta_{-}}-i \pi\right)^{2} \quad \text { for } \tau<1 \\
\eta_{ \pm} & =1 \pm \sqrt{1-\tau}, \quad \tau=4\left(m / m_{h(H)}\right)^{2}
\end{aligned}
$$

The couplings of $h(H)$ with $t \bar{t}, W^{+} W^{-}$, and $H^{+} H^{-}$in the type-I 2HDM are

$$
\begin{aligned}
\xi_{h}^{t} & =\cos \alpha / \sin \beta, \quad \xi_{H}^{t}=\sin \alpha / \sin \beta, \quad \xi_{h}^{W}=\sin (\beta-\alpha), \quad \xi_{H}^{W}=\cos (\beta-\alpha), \\
\xi_{h}^{H^{ \pm}} & =\frac{1}{4 m_{W}^{2} \sin ^{2}(2 \beta)}\left[8 m_{12}^{2} \cos (\alpha+\beta)-\sin (2 \beta)\left(\left(m_{h}^{2}-2 m_{H^{ \pm}}^{2}\right) \cos (\alpha-3 \beta)+\left(2 m_{H^{ \pm}}^{2}+3 m_{h}^{2}\right) \cos (\alpha+\beta)\right)\right], \\
\xi_{H}^{H^{ \pm}} & =\frac{1}{4 m_{W}^{2} \sin (2 \beta)}\left[\left(2 m_{H^{ \pm}}^{2}-m_{H}^{2}\right) \sin (\alpha-3 \beta)+\sin (\alpha+\beta)\left(\frac{4 m_{12}^{2}}{\sin \beta \cos \beta}-2 m_{H^{ \pm}}^{2}-3 m_{H}^{2}\right)\right] .
\end{aligned}
$$

\section{APPENDIX B: CHARGED HIGGS ANALYSIS}

In this appendix, we revisit some of our analyses by considering the effect of a low-mass charged Higgs. We will see that our results will remain more or less unaltered. The independent 2HDM parameters are varied in the following ranges ${ }^{9}$ :

$$
\begin{aligned}
\alpha & =[0, \pi], \quad \tan \beta=[1,10], \quad m_{12}=[0.01,1000] \mathrm{GeV}, \\
M_{A} & =[80,2000] \mathrm{GeV}, \quad M_{H^{ \pm}}=[80,2000] \mathrm{GeV} . \quad(\mathrm{B} 1)
\end{aligned}
$$

As in the type-I 2HDM, couplings decrease as $\tan \beta$ increases, and we have fixed the upper limit on $\tan \beta$ to be 10. We first determine the allowed parameter space by incorporating the following constraints.

(1) Perturbativity: We demand that the Higgs self couplings, i.e., $\lambda_{i}$ and the Yukawa couplings be less than $4 \pi$ for the perturbative expansion to remain valid.

(2) Vacuum stability: This condition ensures that the scalar potential is bounded from below by restricting the $\lambda_{i}$ 's in the ranges

$$
\begin{aligned}
& \lambda_{1,2}>0, \quad \lambda_{3}>-\sqrt{\lambda_{1} \lambda_{2}}, \text { and } \lambda_{3}+\lambda_{4}-\left|\lambda_{5}\right|> \\
& -\sqrt{\lambda_{1} \lambda_{2}} \text { [38]. }
\end{aligned}
$$

(3) Unitarity: This arises from the requirement of unitarity of the scattering amplitudes such that the amplitudes do not grow as the center-of-mass energy increases. The unitary bounds for the $2 \mathrm{HDM}$ can be found in Ref. [39]

(4) $\rho$ parameter $\left(\frac{m_{W}^{2}}{m_{Z}^{2} \cos \theta_{W}}\right)$ : Its value in the $\mathrm{SM}$ is predicted to be unity at tree level (the renormalization scheme is

\footnotetext{
${ }^{9}$ The lower range of $M_{A}$ has been kept the same as that of $M_{H^{ \pm}}$ for simplicity.
}

chosen such that this relation even holds after including higher-order corrections [61]). The experimental prediction of the $\rho$ parameter is in agreement with the SM and constrains the masses of new scalars introduced in the theory [41].

(5) Flavor observables: Although the tree-level FCNCs in the $2 \mathrm{HDM}$ are absent due to the $Z_{2}$ symmetry, the charged scalars can affect these processes through higher-order diagrams. In general, the flavor observables in these models are sensitive to $m_{H^{ \pm}}$ and $\tan \beta$.

(6) Direct charged Higgs searches at LEP: The charged Higgs has been searched for in the channel $e^{+} e^{-} \rightarrow$ $\mathrm{H}^{+} \mathrm{H}^{-}$at LEP. The null observation of the signal has put a lower bound of $80 \mathrm{GeV}$ on the mass of a charged Higgs [62,63]. This bound has been derived assuming $H^{ \pm}$decays only to the $\tau \bar{\nu}$ and $c \bar{s}$ modes. However, in the alignment limit the decay $H^{ \pm} \rightarrow h W^{ \pm}$becomes significant. Hence, the bound on the charged Higgs mass gets relaxed in the regions close to the alignment limit [64].

The effect of the above constraints on the parameter space is shown in Fig. 12. The allowed regions are shown in Figs. 12(a), 12(b), and 12(c) of in the $\left(M_{A}, M_{H^{ \pm}}\right),\left(\alpha, m_{12}\right)$, and $\left(M_{H^{ \pm}}, m_{12}\right)$ planes, respectively. For the type-I model, the bounds from flavor physics are weak and allow almost all values of $m_{H^{ \pm}} \gtrsim 80 \mathrm{GeV}$ for $\tan \beta \gtrsim 2[42,43,65]$. Hence we have not shown the effect in the plot.

The important inferences which we can make from Fig. 12 are as follows:

(1) It can be seen from Fig. 12(a) that there exist upper bounds on the masses of the charged Higgs and the pseudoscalar Higgs. These bounds arise primarily due to the unitarity constraints. Furthermore, bounds from the $\rho$ parameter force the mass of 

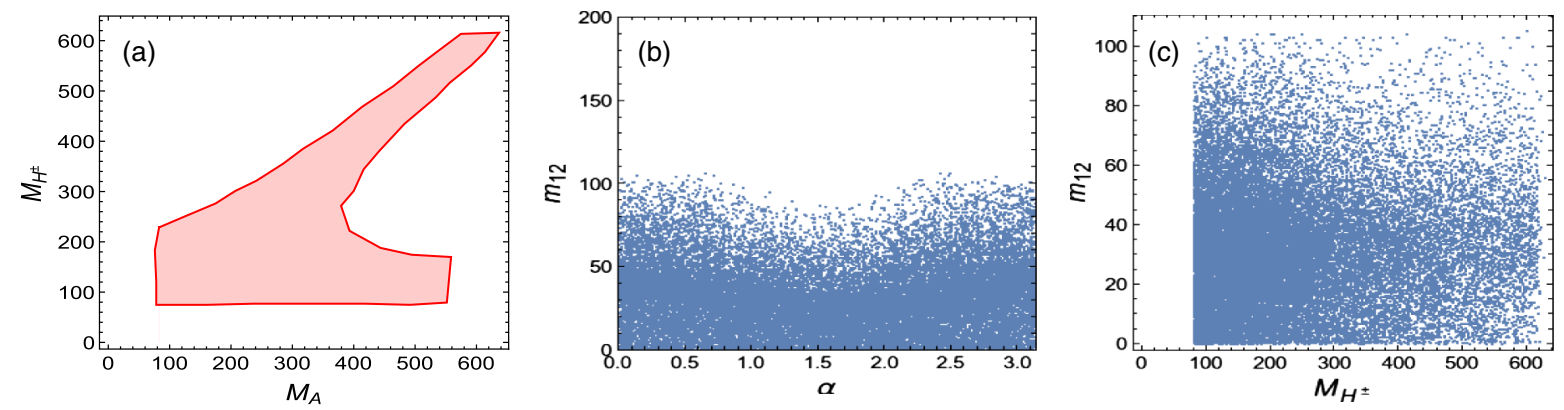

FIG. 12. Allowed ranges in the planes of $\left(M_{A}, M_{H^{ \pm}}\right),\left(\alpha, m_{12}\right)$ and $M_{H^{ \pm}}, m_{12}$ after imposing constraints from perturbativity, vacuum stability, tree-level unitarity, the $\rho$-parameter, LEP and avor data are shown in panels (a), (b) and (c) respectively.
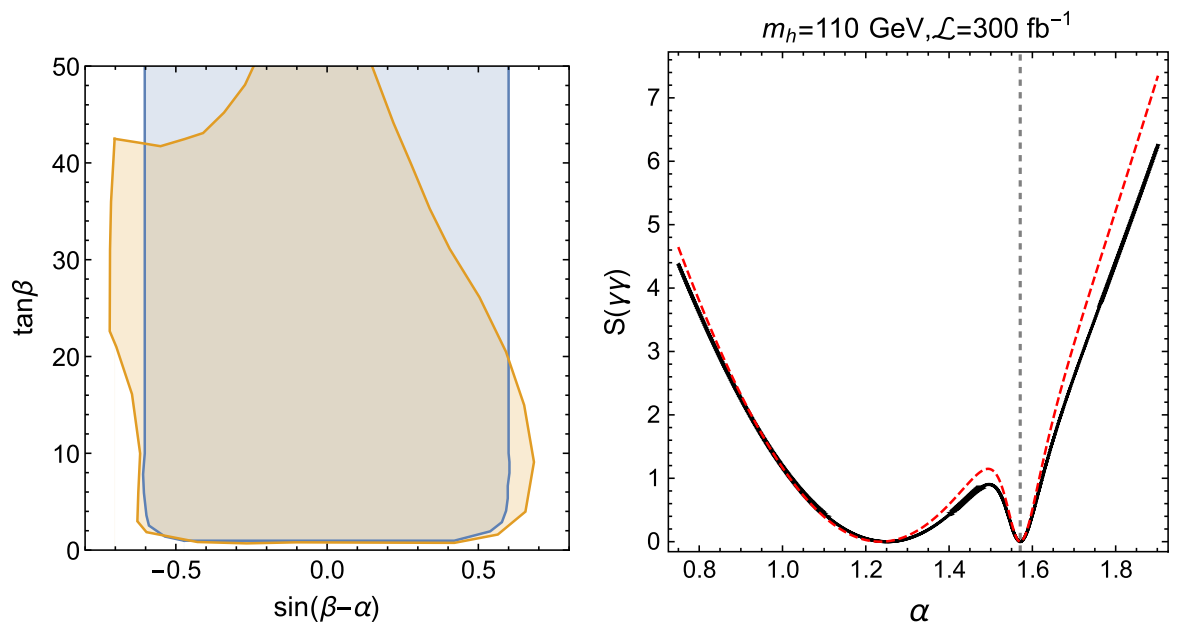

FIG. 13. In the left panel the allowed parameter space from the signal strength data is plotted with and without a charged Higgs. The blue contour shows the allowed parameter space without $H^{ \pm}$and the brown contour is with a $H^{ \pm}$with a mass of $200 \mathrm{GeV}$. In the parameter region we are considering, i.e., $\tan \beta<10$, the effect of adding a charged Higgs is minuscule. In the right panel we show the effect of the charged Higgs on the significance of observing a $\gamma \gamma$ final state. The charged Higgs is found to enhance the significance for large values of $\sin (\beta-\alpha)$. The black (red dashed) line corresponds to the diphoton analysis without (with) the charged Higgs effects.

the pseudoscalar to be approximately equal to that of the charged Higgs for $m_{H^{ \pm}} \gtrsim 200 \mathrm{GeV}$, and for $m_{H^{ \pm}} \lesssim 200 \mathrm{GeV}$ the pseudoscalar mass remains unconstrained.

(2) The $Z_{2}$-symmetry-breaking parameter $m_{12}$ is also restricted to be less than $100 \mathrm{GeV}$ [see Figs. 12(b) and 12(c)]. These bounds arise from the vacuum stability requirements.

(3) The mixing angle $\alpha$ is not constrained at all by any of the above constraints, as can be seen in Fig. 12(c).

After determining the allowed parameter space from theoretical and a few experimental constraints, we proceed to examine the effect of a light charged Higgs on the allowed parameter space from the Higgs signal strength measurements (for earlier analyses of this kind, see Ref. [66]). For illustrative purposes, we have fixed the mass of the charged Higgs to be $200 \mathrm{GeV}$ and $m_{12}^{2}=100 \mathrm{GeV}$. The charged Higgs boson will affect the signal strength measurements through its contribution in $H \rightarrow \gamma \gamma$ decay. It can be seen from Fig. 13 that the deviations in the high- $\tan \beta$ regions are dramatic, while for low $\tan \beta$ the increment in the allowed range of $\sin (\beta-\alpha)$ is slight. Furthermore, for the low-tan $\beta$ regions the LEP measurements are far more constraining (see Fig. 5). Therefore, the allowed parameter space for $\tan \beta<10$ (which is our region of interest) remains the same even after including effects from the low-mass charged Higgs.

A light charged Higgs boson could also affect the significance of observing a light Higgs $h$ in the $\gamma \gamma$ channel. However, it is found that the significance only increases slightly for larger values of $|\sin (\beta-\alpha)|$. The effect is depicted in the right panel of Fig. 13. Although the plot is shown for particular choices of $m_{h}$ and $\tan \beta$, the qualitative result is independent of their values. Hence, the effect of considering a light charged Higgs boson only mildly affects our analyses. 


\section{APPENDIX C: FAT-JET TAGGING TECHNIQUES}

In this appendix we summarize the fat-jet tagging methods for Higgs and top0quark jets [36,37]. We begin with the discussion on the reconstruction of a Higgs fat jet. To start with, we combine all of the momentum fourvectors $\left(j_{i}\right)$ within $\Delta R=0.8$ to form a fat jet $(J)$ using the Cambridge-Aachen algorithm. The fat jets with $p_{T}>$ $200 \mathrm{GeV}$ are considered for further analysis.

(1) The fat jet $(J)$ is broken into two subjets $\left(j_{1}\right.$ and $\left.j_{2}\right)$ and the heavier jet is labeled as $j_{1}$.

(2) The two subjets are considered if the mass of $j_{1}$ has a sufficient mass drop, i.e., $m_{j_{1}}<\mu m_{J}$ and the splitting between two jets defined as $y=\frac{\min \left(p_{T_{1}}, p_{T_{2}}\right)}{\max \left(p_{T_{1}}, p_{T_{2}}\right)}$ is greater than $y_{\text {cut }} \cdot{ }^{10}$ This is a powerful cut to reduce the contaminations due to the QCD background. We have considered $\mu=0.67$ and $y_{\text {cut }}=0.09$ for our analysis [36].

(3) If the previous condition is not satisfied, then $j_{1}$ is identified as $J$ and the procedure is repeated until both of the above conditions are satisfied.

(4) The final jet is considered as the Higgs if both subjets are $b$ tagged and the mass of the filtered ${ }^{11}$ fat jet $\left(m_{J}\right)$ is close to the Higgs mass.
Now we discuss the reconstruction of the top jet. We combine all of the momentum four-vectors $\left(j_{i}\right)$ within $\Delta R=1.2$ to form a fat jet $(J)$ using the Cambridge-Aachen algorithm. The fat jets with $p_{T}>250 \mathrm{GeV}$ are considered for further analysis.

(1) Inside a fat jet, a loose mass-drop criteria is employed such that $J \rightarrow j_{1} j_{2}, \quad m_{j_{2}}<m_{j_{1}}$, and $m_{j_{2}}>0.2 m_{J}$. The splitting takes place iteratively until $m_{j_{1}}>30 \mathrm{GeV}$. A fat jet is retained if it has at least three such subjets.

(2) The three subjets are then filtered with $\Delta R=0.3$ into five subjets. Only those fat jets with a total jet mass close to the top-quark mass are considered. The subjets which reconstruct the top mass are then reclustered into three subjets.

(3) These subjets are then required to satisfy decay kinematics. Among the three pairs of invariant masses with these subjets, two of them are independent (as one of them satisfies the $W$-mass criteria). In a two-dimensional space where the coordinates represent two independent invariant masses, top-like jets represent a thin triangular annulus, whereas the QCD jet is localized in the region of small pairwise invariant mass.

\section{APPENDIX D: CROSS SECTION}

The dependences of the total cross section $(\sigma \times \mathrm{BR})$ on $\alpha$ and $\sin (\beta-\alpha)$ are listed in Table VII and also displayed in Fig. 14.

TABLE VII. The dependences of the total cross section for various processes with respect to the coupling scale factors. The limits where the total cross section vanishes are also listed. The behavior of the total cross section for all four cases with respect to $\alpha$ for $\tan \beta=2$ and 6 is plotted in Fig. 14 .

\begin{tabular}{lccc}
\hline \hline & Total cross section & Parametric dependence & $\begin{array}{c}\text { Limit where the cross } \\
\text { section vanishes }\end{array}$ \\
\hline A & $\sigma(p p \rightarrow h \rightarrow \gamma \gamma)$ & $\left(\frac{\cos \alpha}{\sin \beta}\right)^{2} \times\left|\xi_{h}^{\gamma}\right|^{2} \times \frac{1}{\Gamma_{h}^{\text {ot }}}$ & $\alpha \rightarrow \pi / 2,\left|\xi_{h}^{\gamma}\right| \rightarrow 0$ \\
$\mathrm{~B}$ & $\sigma(p p \rightarrow V h \rightarrow V \gamma \gamma)$ & $\sin (\beta-\alpha)^{2} \times\left|\xi_{h}^{\gamma}\right|^{2} \times \frac{1}{\Gamma_{h}^{\text {Tot }}}$ & $\alpha \rightarrow \beta,\left|\xi_{h}^{\gamma}\right| \rightarrow 0$ \\
$\mathrm{C}$ & $\sigma(p p \rightarrow \bar{t} h \rightarrow \bar{t} b \bar{b})$ & $\left(\frac{\cos \alpha}{\sin \beta}\right)^{4} \times \frac{1}{\Gamma_{h}^{\text {ot }}}$ & $\alpha \rightarrow \pi / 2$ \\
$\mathrm{D}$ & $\sigma(p p \rightarrow V h \rightarrow V b \bar{b})$ & $\sin (\beta-\alpha)^{2} \times\left(\frac{\cos \alpha}{\sin \beta}\right)^{2} \times \frac{1}{\Gamma_{h}^{\text {tot }}}$ & $\alpha \rightarrow \pi / 2, \alpha \rightarrow \beta$ \\
\hline \hline
\end{tabular}

\footnotetext{
${ }^{10}$ This is to ensure that the asymmetric splitting between $j_{1}$ and $j_{2}$ is not too large.

${ }^{11}$ To eliminate underlying events in the fat jet, it is filtered with $R_{\text {filter }}=0.3$ and three hard subjets are retained.
} 

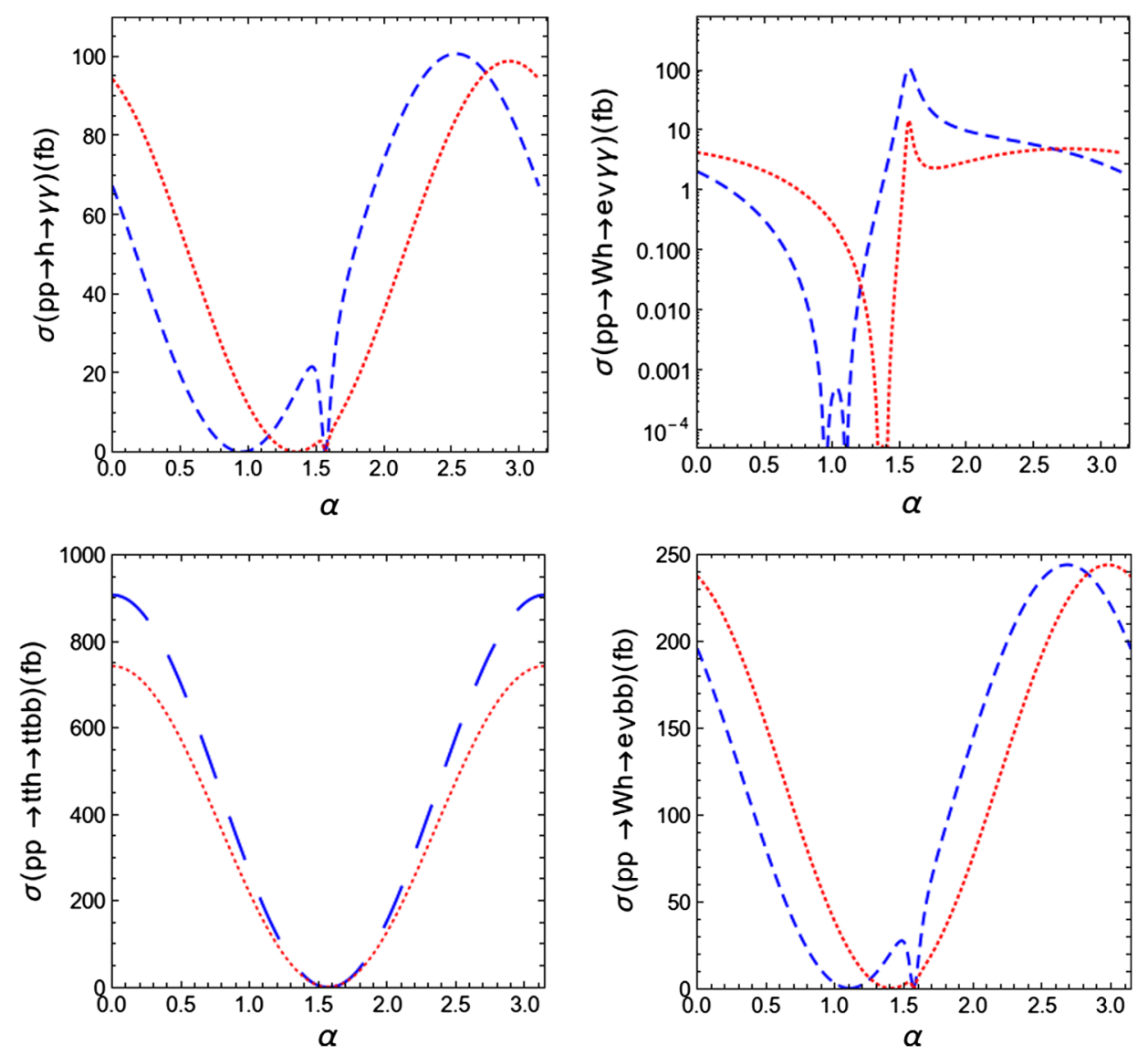

FIG. 14. A representative plot of $(\sigma \times \mathrm{BR})$ for a light Higgs decaying to $\gamma \gamma$ and $b \bar{b}$ for $m_{h}=100 \mathrm{GeV}$. The dashed blue line in corresponds to $\tan \beta=2$, while the red dotted line corresponds to $\tan \beta=6$.

It can be easily seen from the expressions for the cases A and D listed in Table VII that the behavior of the total cross section for $p p \rightarrow h \rightarrow \gamma \gamma$ and $p p \rightarrow W h \rightarrow W b \bar{b}$ becomes identical with respect to $\alpha$ in the large-tan $\beta$ regions. The same can also be verified from the $\tan \beta=6$ line in Fig. 14.

[1] G. Aad et al. (ATLAS Collaboration), Phys. Lett. B 716, 1 (2012).

[2] S. Chatrchyan et al. (CMS Collaboration), Phys. Lett. B 716, 30 (2012).

[3] G. Aad et al. (ATLAS and CMS Collaborations), J. High Energy Phys. 08 (2016) 045.

[4] G. C. Branco, P. M. Ferreira, L. Lavoura, M. N. Rebelo, M. Sher, and J. P. Silva, Phys. Rep. 516, 1 (2012).

[5] G. Aad et al. (ATLAS Collaboration), Phys. Lett. B 726, 120 (2013).

[6] S. Chatrchyan et al. (CMS Collaboration), Phys. Rev. D 89, 092007 (2014).

[7] P. M. Ferreira, R. Santos, M. Sher, and J. P. Silva, Phys. Rev. D 85, 035020 (2012).

[8] A. Celis, V. Ilisie, and A. Pich, J. High Energy Phys. 07 (2013) 053.
[9] S. Chang, S. K. Kang, J.-P. Lee, K. Y. Lee, S. C. Park, and J. Song, J. High Energy Phys. 09 (2014) 101.

[10] B. Dumont, J. F. Gunion, Y. Jiang, and S. Kraml, Phys. Rev. D 90, 035021 (2014).

[11] J. Bernon, J. F. Gunion, Y. Jiang, and S. Kraml, Phys. Rev. D 91, 075019 (2015).

[12] S. Chang, S. K. Kang, J.-P. Lee, and J. Song, Phys. Rev. D 92, 075023 (2015).

[13] J. Bernon, J. F. Gunion, H. E. Haber, Y. Jiang, and S. Kraml, Phys. Rev. D 93, 035027 (2016).

[14] G. Cacciapaglia, A. Deandrea, S. Gascon-Shotkin, S. Le Corre, M. Lethuillier, and J. Tao, J. High Energy Phys. 12 (2016) 068.

[15] D. Goncalves and D. Lopez-Val, Phys. Rev. D 94, 095005 (2016). 
[16] R. Enberg, W. Klemm, S. Moretti, and S. Munir, Phys. Lett. B 764, 121 (2017).

[17] B. Bhattacherjee, M. Chakraborti, A. Chakraborty, U. Chattopadhyay, and D. K. Ghosh, Phys. Rev. D 93, 075004 (2016).

[18] P. Bechtle, H. E. Haber, S. Heinemeyer, O. Stl, T. Stefaniak, G. Weiglein, and L. Zeune, Eur. Phys. J. C 77, 67 (2017).

[19] J. Cao, F. Ding, C. Han, J. M. Yang, and J. Zhu, J. High Energy Phys. 11 (2013) 018.

[20] M. Guchait and J. Kumar, Int. J. Mod. Phys. A 31, 1650069 (2016).

[21] J. F. Gunion and H. E. Haber, Phys. Rev. D 67, 075019 (2003).

[22] C.-W. Chiang and K. Yagyu, J. High Energy Phys. 07 (2013) 160.

[23] O. Eberhardt, U. Nierste, and M. Wiebusch, J. High Energy Phys. 07 (2013) 118.

[24] S. Inoue, M. J. Ramsey-Musolf, and Y. Zhang, Phys. Rev. D 89, 115023 (2014).

[25] A. Celis, V. Ilisie, and A. Pich, J. High Energy Phys. 12 (2013) 095.

[26] N. Craig, J. Galloway, and S. Thomas, arXiv:1305.2424.

[27] C.-Y. Chen, S. Dawson, and M. Sher, Phys. Rev. D 88, 015018 (2013); 88, 039901(E) (2013).

[28] A. Arhrib, P. M. Ferreira, and R. Santos, J. High Energy Phys. 03 (2014) 053.

[29] M. Carena, I. Low, N. R. Shah, and C. E. M. Wagner, J. High Energy Phys. 04 (2014) 015.

[30] J. Bernon, J. F. Gunion, H. E. Haber, Y. Jiang, and S. Kraml, Phys. Rev. D 92, 075004 (2015).

[31] V. Khachatryan et al. (CMS Collaboration), Phys. Rev. D 92, 072010 (2015).

[32] V. Khachatryan et al. (CMS Collaboration), J. High Energy Phys. 10 (2017) 076.

[33] R. Aggleton, D. Barducci, N.-E. Bomark, S. Moretti, and C. Shepherd-Themistocleous, J. High Energy Phys. 02 (2017) 035 .

[34] CMS Collaboration, Technical Report CMS-PAS-HIG-14037, 2015.

[35] G. Aad et al. (ATLAS Collaboration), Phys. Rev. Lett. 113, 171801 (2014).

[36] J. M. Butterworth, A. R. Davison, M. Rubin, and G. P. Salam, Phys. Rev. Lett. 100, 242001 (2008).

[37] T. Plehn and M. Spannowsky, J. Phys. G 39, 083001 (2012).

[38] S. Nie and M. Sher, Phys. Lett. B 449, 89 (1999).

[39] A. G. Akeroyd, A. Arhrib, and E.-M. Naimi, Phys. Lett. B 490, 119 (2000).

[40] G. Bhattacharyya and D. Das, Pramana 87, 40 (2016).
[41] S. Schael et al., Phys. Rep. 427, 257 (2006).

[42] F. Mahmoudi and O. Stal, Phys. Rev. D 81, 035016 (2010).

[43] M. Aoki, S. Kanemura, K. Tsumura, and K. Yagyu, Phys. Rev. D 80, 015017 (2009).

[44] J. F. Gunion, H. E. Haber, G. L. Kane, and S. Dawson, Front. Phys. 80, 1 (2000).

[45] V.D. Barger and R. J. N. Phillips, Collider Physics (CRC Press, Boca Raton, 1987).

[46] S. Dawson et al., arXiv:1310.8361.

[47] S. Schael et al., Eur. Phys. J. C 47, 547 (2006).

[48] T. Aaltonen et al. (CDF Collaboration), Phys. Rev. D 87, 052008 (2013).

[49] ALEPH, DELPHI, L3, and OPAL Collaborations and LEP Higgs Working Group, arXiv:hep-ex/0107030.

[50] DELPHI Collaboration, Eur. Phys. J. C 35, 313 (2004).

[51] CMS Collaboration, Technical Report CMS-PAS-HIG-17013, 2017.

[52] J. Alwall, R. Frederix, S. Frixione, V. Hirschi, F. Maltoni, O. Mattelaer, H. S. Shao, T. Stelzer, P. Torrielli, and M. Zaro, J. High Energy Phys. 07 (2014) 079.

[53] T. Sjostrand, S. Mrenna, and P. Z. Skands, Comput. Phys. Commun. 178, 852 (2008).

[54] T. Sjstrand, S. Ask, J. R. Christiansen, R. Corke, N. Desai, P. Ilten, S. Mrenna, S. Prestel, C. O. Rasmussen, and P.Z. Skands, Comput. Phys. Commun. 191, 159 (2015).

[55] R. D. Ball, V. Bertone, S. Carrazza, L. Del Debbio, S. Forte, A. Guffanti, N. P. Hartland, and J. Rojo (NNPDF Collaboration), Nucl. Phys. B877, 290 (2013).

[56] M. Cacciari, G. P. Salam, and G. Soyez, J. High Energy Phys. 04 (2008) 063.

[57] R. Barate et al., Phys. Lett. B 565, 61 (2003).

[58] P. J. Fox and N. Weiner, arXiv:1710.07649.

[59] U. Haisch and A. Malinauskas, arXiv:1712.06599.

[60] J. F. Gunion, S. Dawson, H. E. Haber, and G. L. Kane, The Higgs Hunter's Guide, Vol. 80 (Brookhaven National Laboratory, Upton, NY, 1989).

[61] D. A. Ross and J. C. Taylor, Nucl. Phys. B51, 125 (1973); B58, 643(E) (1973).

[62] ALEPH, DELPHI, L3, and OPAL Collaborations and LEP Working Group for Higgs Boson Searches, arXiv: hep-ex/0107031.

[63] P. Abreu et al. (DELPHI Collaboration), Phys. Lett. B 507, 89 (2001).

[64] A. Arbey, F. Mahmoudi, O. Stal, and T. Stefaniak, arXiv: 1706.07414.

[65] A. Arbey and F. Mahmoudi, Comput. Phys. Commun. 182, 1582 (2011).

[66] P. Posch, Phys. Lett. B 696, 447 (2011). 